\title{
Nonlinear position and stiffness Backstepping controller for a two Degrees of Freedom pneumatic robot
}

\author{
Nicolas Herzig $^{\mathrm{a}, *}$, Richard Moreau ${ }^{\mathrm{b}}$, Tanneguy Redarce ${ }^{\mathrm{b}}$, Frédéric Abry ${ }^{\mathrm{b}}$, Xavier Brun ${ }^{\mathrm{b}}$ \\ a Dyson School of Design Engineering, Imperial College London, South Kesington, SW7 2AZ, London, United Kingdom \\ ${ }^{\mathrm{b}}$ Laboratoire Ampère UMR CNRS 5005, Université de Lyon, INSA-Lyon, F-69621 Villeurbanne Cedex, France
}

\section{A R T T I C L E I N}

\section{Keywords:}

Backstepping controller design

Damping control

Electropneumatic robot

Nonlinear control

Stiffness control

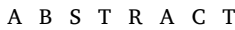

This paper presents an architecture of a 2 Degrees of Freedom pneumatic robot which can be used as a haptic interface. To improve the haptic rendering of this device, a nonlinear position and stiffness controller without force measurement based on a Backstepping synthesis is presented. Thus, the robot can follow a targeted trajectory in Cartesian position with a variable compliant behavior when disturbance forces are applied. An appropriate tuning methodology of the closed-loop stiffness and closed-loop damping of the robot is given to obtain a desired disturbance response. The models, the synthesis and the stability analysis of this controller are described in this paper. Two models are presented in this paper, the first one is an accurate simulation model which describes the mechanical behavior of the robot, the thermodynamics phenomena in the pneumatic actuators, and the servovalves characteristics. The second model is the model used to synthesize the controller. This control model is obtained by simplifying the simulation model to obtain a MIMO strict feedback form. Finally, some simulation and experimental results are given and the controller performances are discussed and compared with a classical linear impedance controller.
\end{abstract}

(C) 2018 The Authors. Published by Elsevier Ltd. This is an open access article under the CC BY license (http://creativecommons.org/licenses/by/4.0/).

\section{Introduction}

Many robotic applications require an interaction between the endeffector of the robot and an uncertain environment. For instance, for human rehabilitation, for haptic interfaces, or for prosthetic devices, human-robot interactions are necessary. When these interactions occur, most of the time, a compliant behavior of the robot is required in order to avoid human injuries or to avoid damaging the robot itself. But on the other hand, these robots have to be stiff for some tasks. Therefore it is necessary to control the stiffness and damping of the robots. To ensure a compliant behavior of a robot, various Variable Stiffness Actuators (VSAs) or Variable Impedance Actuators (VIAs) have been developed during last decades. These actuators allow the equilibrium position and the stiffness to be tuned independently. Van Ham, Sugar, Vanderborght, Hollander, and Lefeber (2009) present a state of the art in the design of VSAs. Most of these actuators are designed with two internal motors and passive compliant elements. An advantage of this design is that the position and stiffness control of the VSA is obtained by controlling the position of two electric motors. The main drawbacks of this kind of VSAs are the cost and the stiffness range. Indeed, these actuator are often expensive because two electric actuators are needed to control one Degree Of Freedom (DOF). The range of the stiffness is also often limited (Huang et al., 2013) due to the use of passive stiffness components.

Another approach to obtain a compliant behavior for the robot is based on control strategies such as stiffness control (Salisbury, 1980), impedance control (Hogan, 1987) or hybrid force position control (Hayati, 1986). Most of these strategies have been developed for electromechanically actuated robots. The disadvantages of the electromechanical actuation are that, in order to implement these control strategies, a force/torque sensor is needed. This sensor is required to measure the environment interaction which implies knowing where this interaction will occur. Moreover, these sensors are often expensive and fragile. If force/torque sensors are not used, the actuators have to be backdrivable which mean reducing gear ratio and, consequently, the torque or force range of the robot.

On the other hand, due to their nonlinear behaviors, pneumatic cylinders were traditionally only use as bi-stable position actuators. The recent development of new servovalves and modern robust nonlinear control laws based on sliding mode and Backstepping allowed the

\footnotetext{
* Corresponding author.

E-mail addresses: n.herzig@imperial.ac.uk (N. Herzig), richard.moreau@insa-lyon.fr (R. Moreau), tanneguy.redarce@insa-lyon.fr (T. Redarce), frederic.abry@insa-lyon.fr (F. Abry), xavier.brun@insa-lyon.fr (X. Brun).
} 
Table 1

Main characteristics of the cylinders.

\begin{tabular}{lll}
\hline $\begin{array}{l}\text { Reference } \\
\text { Notation }\end{array}$ & $\begin{array}{l}\text { DSNU-25-400-PPV-A-Q } \\
\text { cylinder 2 } \\
\text { horizontal }\end{array}$ & $\begin{array}{l}\text { DSNU-25-200-PPV-A } \\
\text { cylinder 1 } \\
\text { vertical }\end{array}$ \\
Stroke & $400 \mathrm{~mm}$ & $200 \mathrm{~mm}$ \\
Piston diameter & $25 \mathrm{~mm}$ & $25 \mathrm{~mm}$ \\
Theoretical force at 6 bar, advancing & $295 \mathrm{~N}$ & $295 \mathrm{~N}$ \\
Theoretical force at 6 bar, retracting & $247 \mathrm{~N}$ & $247 \mathrm{~N}$ \\
Rod geometry & $9 \mathrm{~mm} \times 9 \mathrm{~mm}$ (square) & $\emptyset 10 \mathrm{~mm}$ (circle) \\
\hline
\end{tabular}

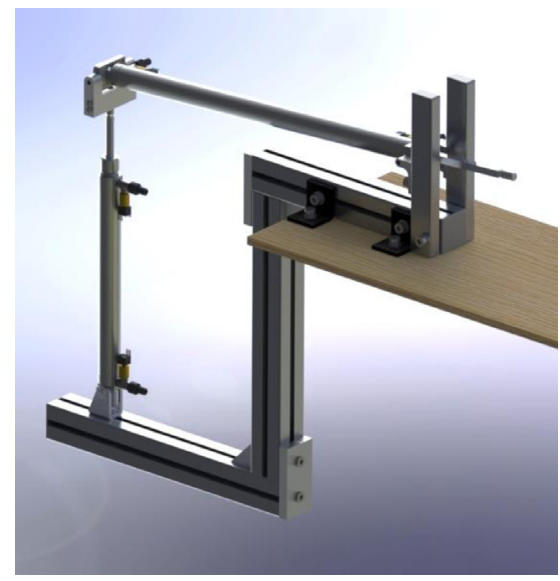

Fig. 1. 2 DOF actuated pneumatic haptic interface.

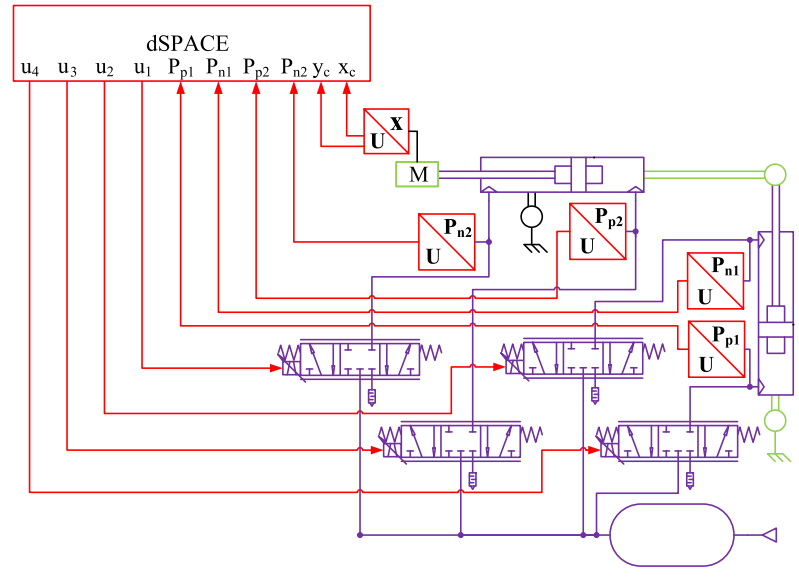

Fig. 2. Hardware architecture of the 2 DOF pneumatic robot. development of position or force controller. Thus, since pneumatic cylinders are inexpensive and have a good power to weight ratio, there has been a recent surge of interest for this technology. If the independent force/stiffness or position/stiffness nonlinear controls of one pneumatic actuator have been addressed in literature (Abry, Brun, Sesmat, Bideaux, \& Ducat, 2015; Shen \& Goldfarb, 2007; Taheri, Case, \& Richer, 2014), the extension of these nonlinear control strategies to multi DOF has not yet been studied. Thus, this article presents an nonlinear position/stiffness control strategy for a 2 DOF pneumatic robot adapted from the Abry et al. position and stiffness controller developed for a pneumatic cylinder (Abry et al., 2015). The synthesis of this controller is based on the Backstepping method and a gain tuning strategy which allows to reach a desired behavior of stiffness and damping.

The presented 2 DOF pneumatic robot is a part of a haptic interface. This haptic device will be used to develop a childbirth simulator. Herzig, Moreau, and Redarce (2014) and Herzig, Moreau, Redarce, Abry, and Brun (2015) give more details about the interest of using this kind of haptic interface to simulate a childbirth delivery.

This paper is structured as follows: In Section 2 the hardware architecture of the 2 DOF actuated robot is given. Then the models used for simulations and for control synthesis are described respectively in Sections 3 and 4. The controller synthesis based on the Backstepping method is described in Section 5. In Section 6 response to an external disturbance force and a strategy to ensure a desired closed-loop stiffness by control gains tuning are discussed. Simulation results and a comparison with a classical linear impedance controller without force sensor are presented in Section 7. Section 8 deals with the experimental results to compare performances of the two controllers for position tracking and disturbance rejection. Finally, Section 9 provides a conclusion and describes future works.

\section{Robot hardware design}

The 2 DOF robot studied in this paper is illustrated in Fig. 1. Its architecture is based on the BirthSIM (Herzig et al., 2014, 2015) design, which is composed of two pneumatic cylinders. The main characteristics of these two cylinders, respectively denoted cylinder 1 and cylinder 2 for the vertical one and the horizontal one, are given in Table 1 . The second cylinder has been chosen with a square rod in order to prevent the inner rotation.

Four Festo MPYE-5-M5-010-B proportional servovalves supply the cylinder chambers. These servovalves control the air mass flow rates which enter or exit the chambers. Their characterization map is given in 3.4. The pressures inside the chambers are measured with Honeywell 40PC100G2A sensors. Moreover, the end-effector Cartesian position and orientation are measured using a Trackstar magnetic tracker. Finally, the controller board is a dSPACE MicroLabBox which is suitable for control prototyping. Fig. 2 illustrates the global hardware architecture of the studied robot.

It has to be noticed that to avoid some usual issues concerning the compression of air in air tubes, the diameter of the air tubes have been chosen small and the length of those tubes have been shortened to the maximum. Indeed, this issue is known for generating delays and also has an impact on the control strategies.

\section{Simulation model}

This section presents the models which are used to test the control law in simulation. To describe the behavior of the robot, mechanical and thermodynamic models have to be defined.

\subsection{Kinematic model}

The Forward Kinematic Model (FKM) and Inverse Kinematic Model (IKM) provide the relations between the location of the end-effector and the joint coordinates. Indeed, the FKM gives the position and orientation of the end-effector as a function of the joint variables whereas the IKM gives the joint variables as a function of the end-effector location. To obtain these models, the Khalil and Kleinfinger method has been used (Khalil \& Kleinfinger, 1986). This method is particularly suitable for robots with closed chains. Fig. 3 presents the kinematic scheme of the studied robot. 


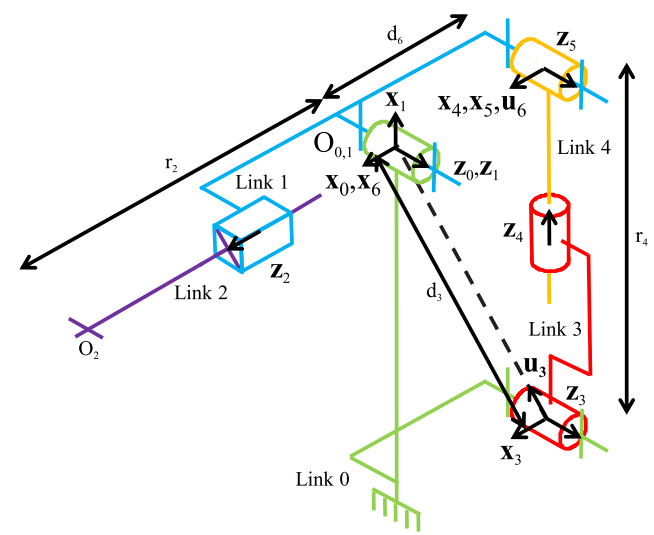

Fig. 3. Kinematic scheme of the 2 DOF pneumatic robot.

The parameters defined with the Khalil and Kleinfinger method are given in Table 2.

As the robot has a closed kinematic chain, joint variables can be separated in three categories. The active joint variables, which are the variables of actuated joints, the passive joint variables and the cut joint variables. Here, the active joint vector $q_{a}$, the passive joint vector $q_{p}$, and the cut joint vector $q_{c}$ are defined as

$q_{a}=\left[\begin{array}{l}r_{4} \\ r_{2}\end{array}\right] \quad q_{p}=\left[\begin{array}{l}\theta_{1} \\ \theta_{3}\end{array}\right] \quad q_{c}=\theta_{5}$.

Then, by solving the constraint equations, the passive joint and cut joint variables are computed as functions of the active joint variables

$\theta_{1}=\gamma_{3}-\arcsin \left(\frac{r_{4}^{2}-d_{3}^{2}-d_{6}^{2}}{2 d_{3} d_{6}}\right)$

$\theta_{3}=-\arcsin \left(\frac{r_{4}^{2}+d_{3}^{2}-d_{6}^{2}}{2 r_{4} d_{3}}\right)$

$\theta_{5}=\arcsin \left(\frac{r_{4}^{2}+d_{6}^{2}-d_{3}^{2}}{2 r_{4} d_{6}}\right)$.

It can be noticed that in the working space of the robot, $\theta_{1}\left(r_{4}\right)$ is bijective.

The FKM can be obtained from the transformation matrix which models the transformation from the $R_{0}$ frame into the $R_{2}$ frame ( $c f$. Fig. 3). As the studied robot is two DOF actuated, only two Cartesian coordinates can be controlled. $x_{c}$ and $y_{c}$ denote the coordinates of $\mathrm{O}_{2}$ (end-effector center) in $R_{0}$ frame. It can be deduced that

$x_{c}=r_{2} \sin \theta_{1}$

$y_{c}=-r_{2} \cos \theta_{1}$

where $\theta_{1}$ is given by (2).

To obtain the IKM, the Paul method (Paul, 1982) has been applied. It leads to the following equations:

$\theta_{1}=\operatorname{atan} 2\left(x_{c},-y_{c}\right)$

$r_{2}=-y_{c} \cos \theta_{1}+x_{c} \sin \theta_{1}$

$r_{4}=\sqrt{d_{3}^{2}+d_{6}^{2}-2 d_{3} d_{6} \sin \left(\theta_{1}-\gamma_{3}\right)}$.

\subsection{Dynamic model}

The dynamic behavior of the two DOF robot presented in this paper can be modeled by the following Newton-Euler formulation:

$\mathbf{M}\left(q_{a}\right) \ddot{q}_{a}+\mathrm{C}\left(q_{a}, \dot{q}_{a}\right)+\mathrm{D}\left(\dot{q}_{a}\right)+\mathrm{G}\left(q_{a}\right)=\mathrm{f}_{\text {pneu }}+\mathrm{f}_{e}$

where $\mathbf{M}$ denotes the symmetric and positive definite inertia matrix, $\mathrm{C}$ is the centrifugal and Coriolis matrix, D is the vector which contains dissipative terms due to friction, $\mathrm{G}$ is the gravity terms vector, $\mathrm{f}_{\text {pneu }}$, and
Table 2

Kinematic parameters of the 2 DOF pneumatic robot.

\begin{tabular}{llllllllll}
\hline$j$ & $a(j)$ & $\sigma$ & $\mu$ & $\mathrm{b}$ & $\mathrm{d}$ & $\mathrm{r}$ & $\gamma$ & $\alpha$ & $\theta$ \\
\hline 1 & 0 & 0 & 0 & 0 & 0 & 0 & 0 & 0 & $\theta_{1}$ \\
2 & 1 & 1 & 1 & 0 & 0 & $r_{2}$ & 0 & $\frac{\pi}{2}$ & 0 \\
3 & 0 & 0 & 0 & 0 & $-d_{3}$ & 0 & $\gamma_{3}$ & 0 & $\theta_{3}$ \\
4 & 3 & 1 & 1 & 0 & 0 & $r_{4}$ & 0 & $-\frac{\pi}{2}$ & 0 \\
5 & 4 & 0 & 0 & 0 & 0 & 0 & 0 & $-\frac{\pi}{2}$ & $\theta_{5}$ \\
6 & 1 & 2 & 0 & 0 & $-d_{6}$ & 0 & $-\frac{\pi}{2}$ & 0 & 0 \\
\hline
\end{tabular}

$\mathrm{f}_{e}$ are respectively the vector of pneumatic forces and external forces generated by the environment or the user in the case of haptic interface.

To simplify the dynamic model and to reduce the parameters which have to be identified, the following assumptions have been taken:

- All the links are assumed to be rigid

- The friction in all revolute joints are neglected compared to the friction of the pistons in cylinders (prismatic joints)

- Only the end-effector mass is taken into account. It is denoted $M$ and is assumed to be a point mass at $O_{2}$. All moments of inertia are neglected.

With these assumptions, $\mathbf{M}$ and $\mathbf{C}$ matrices can be obtained as follows:

$\mathbf{M}\left(q_{a}\right)=\left[\begin{array}{cc}\frac{M r_{2}^{2} r_{4}}{d_{3} d_{6}^{2} \cos \left(\theta_{1}-\gamma_{3}\right) \cos \theta_{5}} & 0 \\ 0 & M\end{array}\right]$

$\mathrm{C}\left(q_{a}, \dot{q}_{a}\right)=$

$\left[\begin{array}{c}M r_{2} \dot{r}_{4} \frac{2 \dot{r}_{2} r_{4}+r_{2} \dot{r}_{4}\left(\frac{r_{4}^{2}\left(r_{4}^{2}-d_{3}^{2}-d_{6}^{2}\right)}{2 d_{3}^{2} d_{6}^{2} \cos ^{2}\left(\theta_{1}-\gamma_{3}\right)}+1\right)}{d_{3} d_{6}^{2} \cos \left(\theta_{1}-\gamma_{3}\right) \cos \theta_{5}} \\ -\frac{M \dot{r}_{4}^{2} r_{4}^{2} r_{2}}{d_{3}^{2} d_{6}^{2} \cos ^{2}\left(\theta_{1}-\gamma_{3}\right)}\end{array}\right]$

where $\cos \left(\theta_{1}-\gamma_{3}\right)$ and $\cos \theta_{5}$ can be deduced from (2)

$\cos \left(\theta_{1}-\gamma_{3}\right)=\sqrt{1-\left(\frac{r_{4}^{2}-d_{3}^{2}-d_{6}^{2}}{2 d_{3} d_{6}}\right)^{2}}$

$\cos \theta_{5}=\sqrt{1-\left(\frac{r_{4}^{2}-d_{3}^{2}+d_{6}^{2}}{2 r_{4} d_{6}}\right)^{2}}$.

The friction model used takes into account the Coulomb friction and the viscous friction. Thus, the parameters $F_{c i}$ and $F_{v i}$ denote respectively the Coulomb and viscous friction parameters of the cylinder $i .^{1}$ These parameters have been identified experimentally with a dedicated test bench. Furthermore, the cylinders used here are not symmetrical so the pneumatic forces have to be modeled as sums of two phenomena. Indeed $F_{\text {pneui }}$ and $F_{\text {pexti }}$ respectively represent the pneumatic force due to the pressure difference between the two chambers and the force applied by the atmospheric pressure on the rod of the cylinder. These two forces are given by the following expressions:

$F_{\text {рпеиi }}=P_{p i} S_{p i}-P_{n i} S_{n i}$

$F_{\text {pexti }}=P_{a t m}\left(S_{p i}-S_{n i}\right)$

where $P_{p i}$ and $P_{n i}$ are the absolute pressures in the P and N chambers, $P_{a t m}$ is the atmospheric pressure, and $S_{p i}$ and $S_{n i}$ are the effective piston areas in chambers $\mathrm{P}$ and $\mathrm{N}$.

Thus, the remaining elements of (5) which describe the dynamic behavior of the robot are given by

${ }^{1}$ In the rest of this paper, the indices $i$ refer to cylinder $i$ with $i \in\{1,2\}$. 


$$
\begin{gathered}
D\left(\dot{q}_{a}\right)=\left[\begin{array}{l}
F_{c 1} \operatorname{sgn}\left(\dot{r}_{4}\right)+F_{v 1} \dot{r}_{4} \\
F_{c 2} \operatorname{sgn}\left(\dot{r}_{2}\right)+F_{v 2} \dot{r}_{2}
\end{array}\right]=\left[\begin{array}{l}
F_{f 1} \\
F_{f 2}
\end{array}\right] \\
\mathrm{G}\left(q_{a}\right)=\left[\begin{array}{c}
-\frac{M g r_{2} \sin \theta_{1}}{d_{6} \cos \theta_{5}} \\
-M g \cos \theta_{1}
\end{array}\right] \\
\mathrm{f}_{\text {pneu }}=\left[\begin{array}{l}
F_{\text {pneu } 1}-F_{\text {pext } 1} \\
F_{\text {pneu } 2}-F_{\text {pext } 2}
\end{array}\right] \\
\mathrm{f}_{e}=\left[\begin{array}{l}
F_{e 1} \\
F_{\text {e2 }}
\end{array}\right]
\end{gathered}
$$

where $g$ is the Earth gravity coefficient, sgn is the sign function, and $F_{e 1}$ and $F_{e 2}$ are respectively the forces exerted by the environment on the piston of cylinder 1 and 2 .

For the sake of clarity, a new couple of variable $y_{1}$ and $y_{2}$ is defined as follows:

$y_{1}=r_{4}-k_{1}$

$y_{2}=r_{2}-k_{2}$

where $k_{1}$ and $k_{2}$ are positive constants. $y_{1} \in\left[-l_{1} / 2 ; l_{1} / 2\right]$ and $y_{2} \in$ $\left[-l_{2} / 2 ; l_{2} / 2\right]$ are respectively the cylinder 1 and 2 piston positions. $l_{1}$ and $l_{2}$ are the strokes of the cylinders.

\subsection{Thermodynamic model}

In this section, the objective is to model the thermodynamic behavior of a cylinder chamber. This kind of model has been addressed in literature (Shearer, 1956). Fig. 4 shows a scheme of a pneumatic cylinder chamber. $P$ denotes the air pressure in the chamber, $V$ is the chamber volume, $T, T_{e n v}$, and $T_{s}$ are respectively the temperature of the air inside the chamber, the temperature of the environment and the temperature of the pressure source. $q_{m}$ is the mass flow rate. It is defined as positive for an entering air flow. $\delta Q$ denotes the heat exchange between the air inside the chamber and the environment. It has to be noticed that the heat exchange between the two chambers is neglected. The assumptions made to model the thermodynamic behavior of the chamber are as follows:

- Air is a perfect gas

- Only the convection is taken into account to model the heat exchanges

- Air leakages are neglected

With these assumptions, the states equations which model the thermodynamic phenomena are

$$
\left\{\begin{array}{c}
\frac{d P}{d t}=\frac{\gamma}{V}\left(r T_{m} q_{m}-P \frac{d V}{d t}\right)+\frac{\gamma-1}{V} h S_{\text {conv }}\left(T_{e n v}-T\right) \\
\frac{d T}{d t}=\frac{T}{P V}\left((1-\gamma) \frac{d V}{d t} P+r\left(\gamma T_{m}-T\right) q_{m}\right. \\
\left.\quad+h S_{\text {conv }}\left(T_{e n v}-T\right)(\gamma-1)\right) \\
T_{m}=T_{s}, \text { for } q>0 \\
T_{m}=T, \text { for } q \leq 0
\end{array}\right.
$$

where $\gamma$ is the heat capacity ratio of a perfect gas, $r$ is the specific gas constant of air, $h$ is the heat transfer coefficient, and $S_{\text {conv }}$ is the convective heat transfer surface. These equations have been written for a chamber. In the case of the studied robot there are four distinct chambers, so the thermodynamic variables will be distinguished with the subscripts $n 1, p 1, n 2$, and $p 2$ which refer to the eponymous chambers.

\subsection{Servovalves model}

In literature, two methods are described to model the servovalves behavior. Both are based on an experimental characterization. The first

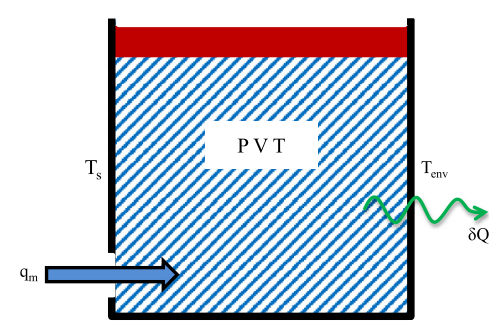

Fig. 4. Model of a cylinder chamber.

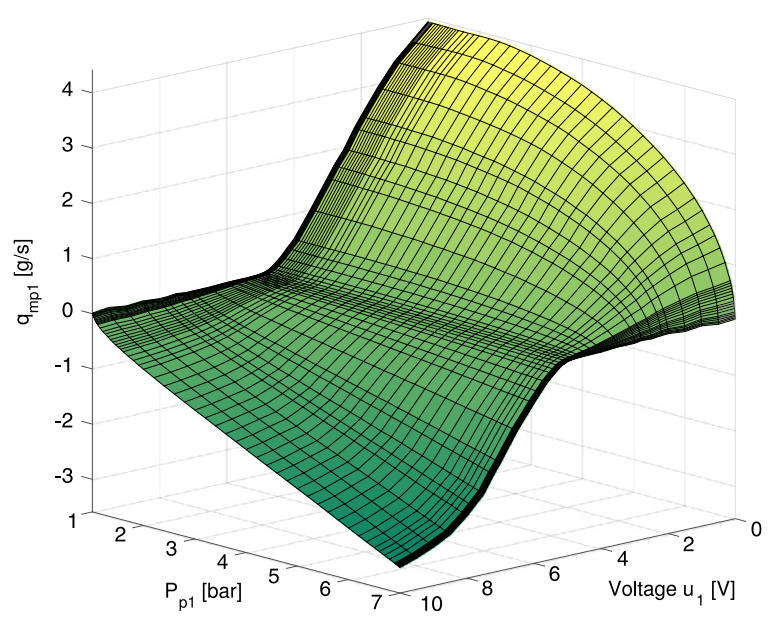

Fig. 5. Characterization map of a Festo MYPE5 servovalve.

one consists in defining a function (most of the time polynomial) fitting the behavior of the servovalve (Bobrow \& McDonell, 1998). The second one consists in an experimental characterization map of the servovalve. To simulate the behavior of the four servovalves of the studied system, the second method has been chosen. Fig. 5. shows the experimental characterization map obtained. It gives the mass flow rate entering the chamber for a given control voltage and a chamber pressure. It can be noticed that once again the behavior of these components is not linear.

\section{Control model}

The model described in the previous section is not adapted to apply the Multi-Input Multi-Output (MIMO) Backstepping method. Indeed, the latter is based on a recursive control design (Freeman \& Kokotovic, 1993; Yao \& Tomizuka, 2001). To apply this method, it is suitable to rewrite the state model in a strict-feedback form. The strict-feedback form MIMO $n$ order system can be described by

$\left\{\begin{array}{r}\dot{x}_{i_{k}}=f_{i_{k}}\left(x_{1}, \ldots, x_{i_{k}}, u_{1}, \ldots, u_{k-1}\right)+g_{i_{k}}\left(x_{1}, \ldots, x_{i_{k}},\right. \\ \left.u_{1}, \ldots, u_{k-1}\right) x_{i_{k}+1}+\delta_{i_{k}, j_{k}} g_{i_{k}, k}\left(x_{1}, \ldots, x_{i_{k}},\right. \\ \left.u_{1}, \ldots, u_{k-1}\right) u_{k} \\ y_{k}=h_{k}\left(x_{1}, \ldots, x_{j_{k}}\right)\end{array}\right.$

where

$$
\begin{gathered}
k \in\{1, \ldots, m\} \\
i_{k} \in\left\{j_{k-1}, \ldots, j_{k}\right\} \\
j_{k}=\sum_{b=1}^{k} n_{b} \\
n=\sum_{k=1}^{m} n_{k}
\end{gathered}
$$


$x_{j_{k-1}}, \ldots, x_{j_{k}}$ are the $n_{k}$ states of the $k$ th block, $u_{1}, \ldots, u_{m}$, and $y_{1}, \ldots, y_{m}$ define the $m$ control inputs and outputs. $\delta_{i_{k}, j_{k}}$ denotes the Kronecker delta. Finally $f, g$, and $h$ denote nonlinear functions.

To write the previous model in a strict-feedback form and then synthesize the Backstepping controller, a change of variables and some model reductions are needed. These reductions can be justified by more assumptions. The first step to reduce the model consists in simplifying the thermodynamics model. Indeed, common assumptions taken to obtain a control model for a pneumatic cylinder are that air inside the chambers of the cylinder follow a polytropic behavior without heat exchange (Andersen, 1967). The second assumption is that the temperature variation of this air is neglected so $T$ is assumed to be constant. These two assumptions lead to replace (11) by

$\left\{\begin{array}{l}\frac{d P_{p i}}{d t}=\frac{k}{S_{p i} L_{p i}\left(y_{i}\right)}\left(r T q_{m p i}-P_{p i} S_{p i} v_{i}\right) \\ \frac{d P_{n i}}{d t}=\frac{k}{S_{n i} L_{n i}\left(y_{i}\right)}\left(r T q_{m n i}+P_{n i} S_{n i} v_{i}\right)\end{array}\right.$

with

$L_{n i}\left(y_{i}\right)=\frac{l_{i}}{2}-y_{i} \quad L_{p i}\left(y_{i}\right)=\frac{l_{i}}{2}+y_{i}$

where $k$ is the polytropic coefficient chosen experimentally, $l_{i}$ is the stroke of the cylinder, $r$ is the specific gas constant for dry air, $T$ is the ambient temperature, $q_{m p i}$ and $q_{m n i}$ are the respective mass flow rates defined as positive entering the chambers $\mathrm{P}$ and $\mathrm{N}$.

Secondly, the A-T transform is applied to change the variables of the system. This transform have been presented by Abry et al. (2015). It can be compared to the Park transform which is used for electric motor control but this transform is adapted to pneumatic actuators control. For each cylinder, the A-T transform introduces two virtual flow rates $q_{m A i}$ and $q_{m T i}$ which respectively are the active and pressurization mass flow rates. These latter can be defined as follows:

$\left[\begin{array}{l}q_{m A i} \\ q_{m T i}\end{array}\right]=\frac{l_{i}}{2}\left[\begin{array}{cc}\frac{1}{L_{p i}\left(y_{i}\right)} & -\frac{1}{L_{n i}\left(y_{i}\right)} \\ \frac{1}{L_{p i}\left(y_{i}\right)} & \frac{1}{L_{n i}\left(y_{i}\right)}\end{array}\right]\left[\begin{array}{l}q_{m p i} \\ q_{m n i}\end{array}\right]$.

The aim of this change of variables is to define two new states $F_{\text {pneui }}$ the pneumatic force given in (8), and $K_{\text {рnеиi }}$ the pneumatic stiffness. The pneumatic stiffness is the position derivative of the pneumatic force free response. Thus

$K_{\text {рпеиi }}=-\frac{d F_{\text {рпеиi }}}{d y_{i}}=k\left(\frac{P_{p i} S_{p i}}{L_{p i}\left(y_{i}\right)}+\frac{P_{n i} S_{n i}}{L_{n i}\left(y_{i}\right)}\right)$.

So the pneumatic model becomes

$$
\left\{\begin{array}{r}
\frac{d F_{\text {рпеиi }}}{d t}=\frac{2 k r T}{l_{i}} q_{m A i}-K_{\text {рпеиi }} v_{i} \\
\frac{d K_{\text {pпеиi }}}{d t}=\frac{A_{1} v_{i} y_{i} K_{\text {pnеиi }}-A_{2} v_{i} F_{\text {pnеиi }}-B_{1, i} y_{i} q_{m A i}}{L_{p i}\left(y_{i}\right) L_{n i}\left(y_{i}\right)} \\
+\frac{B_{2} q_{m T i}}{L_{p i}\left(y_{i}\right) L_{n i}\left(y_{i}\right)}
\end{array}\right.
$$

with

$$
\begin{array}{ll}
A_{1}=2(k+1) & A_{2}=k(k+1) \\
B_{1, i}=\frac{2 k^{2} r T}{l_{i}} & B_{2}=k^{2} r T
\end{array}
$$

On the other hand, to simplify the dynamic model, the first and second time derivatives of $\theta_{1}$ are linearized around the equilibrium point denoted $x_{e}$ where $y_{1}=\sqrt{d_{3}^{2}-d_{6}^{2}}-k_{1}, v_{1}=0$, and $\dot{v}_{1}=0$

$\left.\dot{\theta}_{1} \simeq \frac{\partial \dot{\theta}_{1}}{\partial v_{1}}\right|_{x_{e}} v_{1}+\left.\frac{\partial \dot{\theta}_{1}}{\partial y_{1}}\right|_{x_{e}} y_{1}$

$\dot{\theta}_{1} \simeq-\frac{v_{1}}{d_{6}}$

$\left.\ddot{\theta}_{1} \simeq \frac{\partial \ddot{\theta}_{1}}{\partial \dot{v}_{1}}\right|_{x_{e}} \dot{v}_{1}+\left.\frac{\partial \ddot{\theta}_{1}}{\partial v_{1}}\right|_{x_{e}} v_{1}+\left.\frac{\partial \ddot{\theta}_{1}}{\partial y_{1}}\right|_{x_{e}} y_{1}$

$\ddot{\theta}_{1} \simeq-\frac{\dot{v}_{1}}{d_{6}}$.

It has to be noticed that this simplification is not needed to obtain the MIMO strict-feedback form. This linearization have been done in this paper in order to reduce the size of the equation obtained.

Finally, it is assumed that the weight projection and the centrifugal and Coriolis accelerations on the second cylinder are neglected. Indeed, as this cylinder works around an horizontal position, the weight influence on the dynamic behavior can be neglected. Centrifugal and Coriolis accelerations can be neglected by assuming that the first piston velocity and acceleration stay low. These assumptions lead to rewrite the $\mathbf{M}, \mathrm{C}$, and $\mathrm{G}$ matrices given in (6) and (9) as follows:

$\mathbf{M}\left(q_{a}\right)=\left[\begin{array}{cc}\frac{M r_{2}^{2}}{d_{6}^{2} \cos \theta_{5}} & 0 \\ 0 & M\end{array}\right]$

$\mathrm{C}\left(q_{a}, \dot{q}_{a}\right)=\left[\begin{array}{c}\frac{2 M r_{2} \dot{r}_{2} \dot{r}_{4}}{d_{6}^{2} \cos \theta_{5}} \\ 0\end{array}\right]$

$\mathrm{G}\left(q_{a}\right)=\left[\begin{array}{c}-\frac{M g r_{2} \sin \theta_{1}}{d_{6} \cos \theta_{5}} \\ 0\end{array}\right]$.

By defining the state and control vectors $\mathbf{X}$ and $\mathbf{U}$ as follows:

$\mathbf{X}=\left[\begin{array}{llllllll}x_{1} & x_{2} & x_{3} & x_{4} & x_{5} & x_{6} & x_{7} & x_{8}\end{array}\right]^{T} \quad \mathbf{U}=\left[\begin{array}{llll}u_{1} & u_{2} & u_{3} & u_{4}\end{array}\right]^{T}$

where

$x_{1}=y_{2} \quad x_{5}=y_{1} \quad u_{1}=q_{m A 2}$

$x_{2}=v_{2} \quad x_{6}=v_{1} \quad u_{2}=q_{m T 2}$

$x_{3}=F_{\text {рпеи } 2} \quad x_{7}=F_{\text {рпеи } 1} \quad u_{3}=q_{m A 1}$

$x_{4}=K_{\text {рпеи } 2} x_{8}=K_{\text {рпеи } 1} u_{4}=q_{m T 1}$.

The state model can be written

$\left\{\begin{array}{l}\dot{x}_{1}=x_{2} \\ \dot{x}_{2}=f_{2}\left(x_{1}, x_{2}\right)+g_{2} x_{3} \\ \dot{x}_{3}=g_{3}\left(x_{2}\right) x_{4}+g_{3,1} u_{1} \\ \dot{x}_{4}=f_{4}\left(x_{1}, x_{2}, x_{3}, x_{4}, u_{1}\right)+g_{4,2}\left(x_{1}\right) u_{2} \\ \dot{x}_{5}=x_{6} \\ \dot{x}_{6}=f_{6}\left(x_{1}, x_{2}, x_{5}, x_{6}\right)+g_{6}\left(x_{1}, x_{5}\right) x_{7} \\ \dot{x}_{7}=g_{7}\left(x_{6}\right) x_{8}+g_{7,3} u_{3} \\ \dot{x}_{8}=f_{8}\left(x_{5}, x_{6}, x_{7}, x_{8}, u_{3}\right)+g_{8,4}\left(x_{5}\right) u_{4}\end{array}\right.$.

It has to be noticed that the forces $F_{e 1}$ and $F_{e 2}$ are not taken into account in the control model. Indeed these forces are defined as disturbances and they are not measured. With these assumptions the state model given in (21) respects the MIMO strict feedback form introduced in (12), so the control synthesis by Backstepping method is now applicable.

\section{Controller synthesis}

The model obtained previously is now in a strict feedback form. The Backstepping method can be, therefore, applied to synthesize the control laws. The presented method is based on Abry et al. works (Abry et al., 2015) but has been adapted to the 2 DOF robot presented in Section 2. 
The four virtual mass flow rates are the control inputs. The two active mass flow rates $q_{m A 1}$ and $q_{m A 2}$ will be designed to track the desired position of the pistons $y_{d 1}$ and $y_{d 2}$ respectively for cylinder 1 and 2. To define the trajectory, the derivatives of these positions are needed, thus $y_{d 1}$ and $y_{d 2}$ are required to be $C^{3}$ function of time. The time derivatives of $y_{d 1}$ and $y_{d 2}$ are defined as follows:

$\frac{d y_{d 1}}{d t}=v_{d 1} \frac{d^{2} y_{d 1}}{d t^{2}}=a_{d 1} \frac{d^{3} y_{d 1}}{d t^{3}}=j_{d 1}$

$\frac{d y_{d 2}}{d t}=v_{d 2} \frac{d^{2} y_{d 2}}{d t^{2}}=a_{d 2} \frac{d^{3} y_{d 2}}{d t^{3}}=j_{d 2}$.

The two pressurization mass flow rates $q_{m T 1}$ and $q_{m T 2}$ will be designed to track respectively the desired pneumatic stiffnesses $K_{\text {pneud } 1}$ and $K_{\text {pneud } 2}$ trajectories, this two reference trajectories must be $C^{1}$ functions of time.

\subsection{Cylinder 2 position tracking}

Step 1: The cylinder 2 position tracking error $z_{1}$ is defined as

$z_{1}=y_{2}-y_{d 2}$.

The open loop dynamics of $z_{1}$ is given by

$\dot{z}_{1}=v_{2}-v_{d 2}$

where $v_{2}$ is seen as a virtual input. The latter is chosen as

$v_{2}^{*}=v_{d 2}-C_{1} z_{1}$

where $C_{1}$ is a strictly positive constant.

Step 2: the cylinder 2 velocity tracking error $z_{2}$ is defined as

$z_{2}=v_{2}-v_{2}^{*}$.

The closed-loop dynamics of the cylinder 2 position error and the openloop dynamics of the velocity error are respectively

$\dot{z}_{1}=z_{2}-C_{1} z_{1}$

$\dot{z}_{2}=\frac{F_{\text {рпеи } 2}-F_{\text {pext } 2}-F_{f 2}}{M}-a_{d 2}+C_{1} \dot{z}_{1}$

where $F_{\text {pneu } 2}$ is assumed to be a virtual input. It is chosen as

$$
\begin{array}{r}
F_{\text {pneu } 2}^{*}=F_{\text {pext } 2}+F_{f 2}+M\left(a_{d 2}+z_{1}\left(C_{1}^{2}-1\right)\right. \\
\left.-z_{2}\left(C_{1}+C_{2}\right)\right)
\end{array}
$$

where $C_{2}$ is a strictly positive constant.

Step 3: the cylinder 2 pneumatic force tracking error $z_{3}(t)$ and its integral are defined as

$z_{3}=F_{\text {рпеи } 2}-F_{\text {рпеи } 2}^{*} \quad z_{3 i}=\int z_{3} d t$

So the closed-loop dynamic of $z_{2}$ error and the open-loop dynamic of $z_{3}$ are given by

$$
\begin{array}{r}
\dot{z}_{2}=\frac{z_{3}}{M}-z_{1}-C_{2} z_{2} \\
\dot{z}_{3}=\dot{F}_{\text {рпеи } 2}-b_{2} \frac{F_{\text {pпеи } 2}-F_{\text {pext } 2}-F_{f 2}}{M}-M\left(j_{d 2}\right. \\
+\left(C_{1}^{2}-1\right)\left(z_{2}-C_{1} z_{1}\right)-\left(\frac{z_{3}}{M}-z_{1}\right. \\
\left.\left.\quad-C_{2} z_{2}\right)\left(C_{1}+C_{2}\right)\right) .
\end{array}
$$

The first real control input $q_{m A 2}$ is designed as follows:

$q_{m A 2}=f_{0}+f_{1} z_{1}+f_{2} z_{2}+f_{3} z_{3}+f_{4} z_{3 i}$ with

$f_{0}=\frac{l_{2}\left(b_{2}\left(F_{\text {pneu } 2}-F_{\text {pext } 2}-F_{f 2}\right)+M K_{\text {pпеи } 2} v_{2}\right)}{2 M k r T}$

$f_{1}=\frac{M l_{2}\left(2 C_{1}+C_{2}-C_{1}^{3}\right)}{2 k r T}$

$$
+\frac{l_{2} M j_{d 2}}{2 k r T}
$$

$f_{2}=\frac{l_{2}\left(M^{2}\left(C_{1}^{2}+C_{1} C_{2}+C_{2}^{2}-1\right)-1\right)}{2 M k R T}$

$f_{3}=-\frac{l_{2}\left(C_{1}+C_{2}+C_{3}\right)}{2 k r T}$

$f_{4}=-\frac{K_{i 3} l_{2}}{2 k r T}$

where $C_{3}$ and $K_{i 3}$ are strictly positive constants. $z_{3 i}$ error is added in

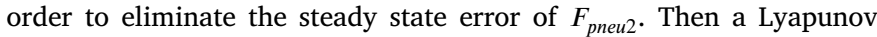
function candidate, denoted $V_{1}$, is chosen as

$V_{1}=\frac{z_{1}^{2}}{2}+\frac{z_{2}^{2}}{2}+\frac{z_{3}^{2}}{2}+K_{i 3} \frac{z_{3 i}^{2}}{2}$.

Using (27), (30) and (31), the derivative can be computed

$\dot{V}_{1}=-C_{1} z_{1}^{2}-C_{2} z_{2}^{2}-C_{3} z_{3}^{2}$.

\subsection{Cylinder 2 pneumatic stiffness tracking}

Step 4: the cylinder 2 pneumatic stiffness error $z_{4}$ is defined as

$z_{4}=K_{\text {рпеи } 2}-K_{\text {рпеид } 2}$.

The open-loop dynamic of this error is given by

$$
\begin{array}{r}
\dot{z}_{4}=\frac{A_{1} v_{2} y_{2} K_{\text {pneu } 2}-A_{2} v_{2} F_{\text {pneu } 2}-B_{1,2} y_{2} q_{m A 2}}{L_{p 2}\left(y_{2}\right) L_{n 2}\left(y_{2}\right)} \\
+\frac{B_{2} q_{m T 2}}{L_{p 2}\left(y_{2}\right) L_{n 2}\left(y_{2}\right)}-\dot{K}_{\text {pneud } 2} .
\end{array}
$$

The second real input $q_{m T 2}$ is designed as follows:

$$
\begin{array}{r}
q_{m T 2}=\frac{\dot{K}_{\text {рпеи } 2}-C_{4} z_{4}}{B_{2}} \\
L_{p i}\left(y_{i}\right) L_{n i}\left(y_{i}\right)+\frac{A_{2} v_{2} F_{\text {рпеи } 2}}{B_{2}} \\
-\frac{A_{1} v_{2} y_{2} K_{\text {pпеи } 2}}{B_{2}}+\frac{2 y_{2} q_{m A 2}}{l_{2}}
\end{array}
$$

where $C_{4}$ is a strictly positive constant. Due to the particular form of the model, it can be noticed that this input depends on $q_{m A 2}$ the previous input designed during the first step. Then a second Lyapunov function candidate $V_{2}$ is chosen as

$V_{2}=\frac{z_{4}^{2}}{2}$.

By substituting $q_{m T 2}$ with the expression obtained in (36), the time derivative of $V_{2}$ is

$\dot{V}_{2}=-C_{4} z_{4}^{2}$

\subsection{Cylinder 1 position tracking}

Step 5: As for the cylinder 2 the cylinder 1 position and velocity errors, respectively $z_{5}$ and $z_{6}$ are defined as

$z_{5}=y_{1}-y_{d 1}$

$z_{6}=v_{1}-v_{1}^{*}$

where $v_{1}^{*}$ is a virtual control. The open-loop dynamic of $z_{5}$ is given by the equation

$\dot{z}_{5}=v_{1}-v_{d 1}$ 
Then the virtual control $v_{1}^{*}$ is designed as

$v_{1}^{*}=v_{d 1}-C_{5} z_{5}$

where $C_{5}$ is a strictly positive constant. Thus the closed-loop dynamic of the cylinder 1 piston position obtained is given by

$\dot{z}_{5}=z_{6}-C_{5} z_{5}$.

Step 6: The tracking error dynamic of the cylinder 1 piston position can be written as follows:

$$
\begin{array}{r}
\dot{z}_{6}=\frac{d_{6}}{y_{2}+k_{2}}\left(g \sin \theta_{1}-\frac{d_{6} \cos \theta_{5}}{M\left(y_{2}+k_{2}\right)}\left(F_{f 1}+F_{\text {pext } 1}\right.\right. \\
\left.\left.-F_{\text {pneu } 1}\right)\right)-\frac{v_{1} v_{2}}{y_{2}+k_{2}}-a_{d 1}+C_{5} \dot{z}_{5}
\end{array}
$$

where $F_{\text {рпеи } 1}$ is assumed to be a virtual input and is chosen as

$$
\begin{aligned}
F_{\text {pneu } 1}^{*} & =F_{\text {pext } 1}+F_{f 1}+\frac{M\left(y_{2}+k_{2}\right)^{2}}{d_{6}^{2} \cos \theta_{5}}\left(a_{d 1}+\left(C_{5}^{2}\right.\right. \\
& \left.-1) z_{5}-\left(C_{5}+C_{6}\right) z_{6}\right)+\frac{M v_{1} v_{2}\left(y_{2}+k_{2}\right)}{d_{6}^{2} \cos \theta_{5}} \\
& -\frac{M g \sin \theta_{1}\left(y_{2}+k_{2}\right)}{d_{6} \cos \theta_{5}}
\end{aligned}
$$

where $C_{6}$ is a strictly positive constant.

Step 7: the pneumatic effort tracking error $z_{7}$ is defined as

$z_{7}=F_{\text {pпеи } 1}-F_{\text {pneu } 1}^{*}$.

The closed-loop dynamic of $z_{6}$ and respectively the open-loop of $z_{7}$ are given by

$\dot{z}_{6}=\frac{d_{6}^{2} \cos \theta_{5}}{M\left(y_{2}+k_{2}\right)^{2}} z_{7}-z_{5}-C_{6} z_{6}$

$\dot{z}_{7}=\dot{F}_{\text {pneu } 1}-\dot{F}_{\text {pneu } 1}^{*}$.

To simplify the expression, $\dot{F}_{p n e u 1}^{*}$ is not given here but in Appendix A.

The design of the third real control $q_{m A 1}$ is given by

$$
\begin{array}{r}
q_{m A 1}=\frac{l_{1}}{2 k r T}\left(\dot{F}_{\text {pпеи } 1}^{*}+K_{\text {pnеu } 1} v_{1}-C_{7} z_{7}-K_{i 7} z_{7 i}\right. \\
\left.-\frac{d_{6} \cos \theta_{5}}{M\left(y_{2}+k_{2}\right)^{2}} z_{6}\right)
\end{array}
$$

where $C_{7}$ and $K_{i 7}$ are strictly positive constants. $z_{7 i}$ is the integral of $z_{7}$ error and is given by $z_{7 i}=\int z_{7} d t$. This integral error is added in order to eliminate the steady state error of $F_{\text {pneu } 1}$. Then a Lyapunov function candidate denoted $V_{3}$ is chosen as

$V_{3}=C_{5} \frac{z_{5}^{2}}{2}+C_{6} \frac{z_{6}^{2}}{2}+C_{7} \frac{z_{7}^{2}}{2}+K_{i 7} \frac{z_{7 i}^{2}}{2}$.

By using the results obtained in (42), (46) and (47) The derivative of this function is

$\dot{V}_{3}=-C_{5} z_{5}^{2}-C_{6} z_{6}^{2}-C_{7} z_{7}^{2}$.

\subsection{Cylinder 1 pneumatic stiffness tracking}

Step 8: The cylinder 1 pneumatic stiffness tracking error $z_{8}$ is defined as

$z_{8}=K_{\text {pneu } 1}-K_{\text {pneud } 1}$.

The open-loop dynamic of $z_{8}$ is given by its time derivative

$$
\begin{array}{r}
\dot{z}_{8}=\frac{A_{1} v_{1} y_{1} K_{\text {pneи } 1}-A_{2} v_{1} F_{\text {pneu } 1}-B_{1,1} y_{1} q_{m A 1}}{L_{p 1}\left(y_{1}\right) L_{n 1}\left(y_{1}\right)} \\
+\frac{B_{2} q_{m T 1}}{L_{p 1}\left(y_{1}\right) L_{n 1}\left(y_{1}\right)}-\dot{K}_{p \text { nеu } 1} .
\end{array}
$$

Then the last real control is chosen as follows:

$$
\begin{array}{r}
q_{m T 1}=\frac{\dot{K}_{\text {pпеи } 1}-C_{8} z_{8}}{B_{2}} L_{p 1}\left(y_{1}\right) L_{n 1}\left(y_{1}\right)+\frac{A_{2} v_{1} F_{\text {pneи } 1}}{B_{2}} \\
-\frac{A_{1} v_{1} y_{1} K_{\text {pneи } 1}}{B_{2}}-\frac{2 y_{1} q_{\text {ma } 1}}{l_{1}}
\end{array}
$$

where $C_{8}$ is a strictly positive constant. Finally a last Lyapunov function is chosen

$V_{4}=\frac{z_{8}^{2}}{2}$

Using (52), its derivative can be computed

$\dot{V}_{4}=-C_{8} z_{8}^{2}$

\subsection{Stability analysis}

Using the Backstepping method, the four controls $q_{m A 1}, q_{m T 1}, q_{m A 2}$, and $q_{m T 2}$ have been designed in order to ensure the closed-loop global asymptotic convergence of the positions and pneumatic stiffnesses of cylinders 1 and 2 tracking errors. Indeed, the following Lyapunov candidate is chosen for the whole system:

$V=V_{1}+V_{2}+V_{3}+V_{4}$.

It can be noticed that $V$ is positive definite. Its time derivative can be deduced from (33), (38), (49), (54)

$\dot{V}=-\sum_{i=1}^{8} C_{i} z_{i}^{2}$.

As the time derivative of $V$ is negative definite the Lyapunov theory ensures the global asymptotic convergence of the system.

\section{Disturbance rejection and closed-loop stiffness}

The controller synthesis method has been chosen because Abry et al. have shown that the tuning of some gains allows to control the system disturbance response (Abry et al., 2015). Indeed, it is possible to tune the closed-loop stiffness and damping of each actuator by adapting the control gains. It is important to distinguish the pneumatic stiffness and the closed-loop stiffness. Indeed, the pneumatic stiffness described in (15) is a state of the system. This state represents the actuator natural tendency to counteract an external effort applied on the piston. This response depends on the piston position but also on the chambers pressures. On the other hand, the closed-loop stiffness describe how the controlled actuator will react to a position error due to an external force or disturbance. Thus for the two pneumatic actuators the closed-loop stiffnesses can be expressed as

$K_{c l 2}=\frac{d F_{e 2}}{d z_{1}}$

$K_{c l 1}=\frac{d F_{e 1}}{d z_{4}}$

with $z_{1}=y_{1}-y_{d 1}$ et $z_{4}=y_{2}-y_{d 2} . F_{e 1}$ and $F_{e 2}$ are respectively the external or disturbance forces apply on cylinder 1 and 2 . By assuming that the disturbance rejection is quasi-static, the external forces become

$$
\begin{aligned}
& F_{e 2}=-\left(F_{\text {pпеи } 2}-F_{\text {pext } 2}-F_{f 2}\right) \\
& F_{e 1}=F_{f 1}-\frac{M\left(y_{2}+k_{2}\right)}{d_{6} \cos \theta_{5}}\left(g \sin \theta_{1}-\frac{v_{1} v_{2}}{d_{6}}\right) \\
&-F_{\text {pпеи } 1}+F_{\text {pext } 1} .
\end{aligned}
$$

Then, thanks to the integral actions introduced in (31) and (47) the pneumatic efforts $z_{3}$ et $z_{7}$ converge quickly to zero. Therefore, it can 
be assumed that $F_{\text {pneu } 1}=F_{\text {pneu } 1}^{*}$ and $F_{\text {pneu } 2}=F_{\text {pneu } 2}^{*}$. Thus, (57) can be expressed as follows:

$$
\begin{gathered}
K_{c l 2}=-M \frac{d\left(a_{d 2}+z_{1}\left(C_{1}^{2}-1\right)-z_{2}\left(C_{1}+C_{2}\right)\right)}{d z_{1}} \\
K_{c l 1}=-\frac{d\left(\frac{M\left(y_{2}+k_{2}\right)^{2}}{d_{6}^{2} \cos \theta_{5}}\left(a_{d 1}+\left(C_{5}^{2}-1\right) z_{5}\right)\right)}{d z_{5}} \\
+\frac{d\left(\frac{M\left(y_{2}+k_{2}\right)^{2}}{d_{6}^{2} \cos \theta_{5}}\left(C_{5}+C_{6}\right) z_{6}\right)}{d z_{5}} .
\end{gathered}
$$

By taking into account that $z_{2}=v_{2}-v_{d 2}+C_{1} z_{1}$ et $z_{6}=v_{1}-v_{d 1}+C_{5} z_{5}$, and neglecting the variation of $\cos \theta_{5}$ in $K_{c l 2}$

$\begin{aligned} K_{c l 2} & =M\left(C_{1} C_{2}+1\right) \\ K_{c l 1} & =\frac{M\left(y_{2}+k_{2}\right)^{2}}{d_{6}^{2} \cos \theta_{5}}\left(C_{5} C_{6}+1\right) .\end{aligned}$

Identically, the closed-loop dampings of the two cylinders can be defined as

$B_{c l 2}=\frac{d F_{e 2}}{d \tilde{v}_{2}}$

$B_{c l 1}=\frac{d F_{e 1}}{d \tilde{v}_{1}}$

with $\tilde{v}_{i}=v_{i}-v_{d i}$. After simplifications, the closed-loop dampings are given by

$B_{c l 2}=M\left(C_{1}+C_{2}\right)$

$B_{c l 1}=\frac{M\left(y_{2}+k_{2}\right)^{2}}{d_{6}^{2} \cos \theta_{5}}\left(C_{5}+C_{6}\right)$.

By solving the Eqs. (60) and (62) for desired closed-loop stiffnesses and dampings, $C_{1}, C_{2}, C_{5}$, and $C_{6}$ can be computed

$C_{1}=\frac{B_{c l 2}+\sqrt{B_{c l 2}^{2}-4 M\left(K_{c l 2}-M\right)}}{2 M}$

$C_{2}=\frac{B_{c l 2}-\sqrt{B_{c l 2}^{2}-4 M\left(K_{c l 2}-M\right)}}{2 M}$

$C_{5}=d_{6}^{2} \cos \theta_{5} \frac{B_{c l 1}+\sqrt{\Delta}}{2 M\left(y_{2}+k_{2}\right)^{2}}$

$C_{6}=d_{6}^{2} \cos \theta_{5} \frac{B_{c l 1}-\sqrt{\Delta}}{2 M\left(y_{2}+k_{2}\right)^{2}}$

with

$\Delta=B_{c l 1}^{2}-4 \frac{M\left(y_{2}+k_{2}\right)^{2}}{d_{6}^{2} \cos \theta_{5}}\left(K_{c l 1}-\frac{M\left(y_{2}+k_{2}\right)^{2}}{d_{6}^{2} \cos \theta_{5}}\right)$.

It can be noticed that the values of $C_{1}$ and $C_{2}$, and respectively $C_{5}$ and $C_{6}$ are interchangeable. Moreover, to ensure stability $C_{1}, C_{2}, C_{5}$, and $C_{6}$ have to be strictly positive. Therefore, the following condition are necessary

$K_{c l 1}>\frac{M\left(y_{2}+k_{2}\right)^{2}}{d_{6}^{2} \cos \theta_{5}}$

$K_{c l 2}>M$

$B_{c l 1} \geq 2 \sqrt{\frac{M\left(y_{2}+k_{2}\right)^{2}}{d_{6}^{2} \cos \theta_{5}}\left(K_{c l 1}-\frac{M\left(y_{2}+k_{2}\right)^{2}}{d_{6}^{2} \cos \theta_{5}}\right)}$

$B_{c l 2} \geq 2 \sqrt{M\left(K_{c l 2}-M\right)}$.

The presented method is used to tune some of the control gains in order to set the closed-loop stiffness and damping of each actuator. Most of the time, the part which will interact with the environment is the endeffector. So, in order to tune the equivalent stiffness of the end-effector in Cartesian space $K_{c l x}$ and $K_{c l y}$, the closed-loop stiffnesses $K_{c l 1}$ and $K_{c l 2}$

\begin{tabular}{|c|c|c|c|c|c|}
\hline \multicolumn{6}{|c|}{ Mechanical parameters } \\
\hline$M$ & 2 & $\mathrm{~kg}$ & $S_{p 1}$ & $4.91 \times 10^{-4}$ & $\mathrm{~m}^{2}$ \\
\hline$g$ & 9.81 & $\mathrm{~m} \cdot \mathrm{s}^{-2}$ & $S_{n 1}$ & $4.12 \times 10^{-4}$ & $\mathrm{~m}^{2}$ \\
\hline$F_{c 1}$ & 15 & $\mathrm{~N}$ & $S_{p 2}$ & $4.91 \times 10^{-4}$ & $\mathrm{~m}^{2}$ \\
\hline$F_{v 1}$ & 50 & N.s.m ${ }^{-1}$ & $S_{n 2}$ & $4.10 \times 10^{-4}$ & $\mathrm{~m}^{2}$ \\
\hline$F_{v 2}$ & 50 & N.s.m ${ }^{-1}$ & $d_{3}$ & $644 \times 10^{-3}$ & $\mathrm{~m}$ \\
\hline$F_{c 2}$ & 15 & $\mathrm{~N}$ & $d_{6}$ & $477.5 \times 10^{-3}$ & $\mathrm{~m}$ \\
\hline$l_{1}$ & 0.2 & $\mathrm{~m}^{2}$ & $k_{1}$ & $457 \times 10^{-3}$ & $\mathrm{~m}$ \\
\hline$l_{2}$ & 0.4 & $\mathrm{~m}^{2}$ & $k_{2}$ & $250 \times 10^{-3}$ & $\mathrm{~m}$ \\
\hline$\gamma_{5}$ & 0.7401 & $\mathrm{rad}$ & & & \\
\hline \multicolumn{6}{|c|}{ Thermodynamic parameters } \\
\hline$r$ & 287 & $\mathrm{~J} . \mathrm{kg}^{-1} \cdot \mathrm{K}^{-1}$ & $T_{e n v}$ & 298 & $\mathrm{~K}$ \\
\hline$\gamma$ & 1.4 & SI & $P_{a t m}$ & $10^{5}$ & $\mathrm{~Pa}$ \\
\hline
\end{tabular}

Table 3

Robot parameters.

have to be computed. To do so it is necessary to introduce the kinematic Jacobian matrix denoted $\mathbf{J}\left(y_{1}, y_{2}\right)$.

$\left[\begin{array}{l}d x_{c} \\ d y_{c}\end{array}\right]=\mathbf{J}\left(y_{1}, y_{2}\right)\left[\begin{array}{l}d y_{1} \\ d y_{2}\end{array}\right]$

where $d x_{c}$ and $d y_{c}$ are respectively the Cartesian end-effector positions $x_{c}$ and $y_{c}$ differentials.

By assuming that the only disturbances applied on the cylinders are the result of a disturbance applied on the end-effector, because of the force velocity duality it comes

$\left[\begin{array}{c}F_{e 1} \\ F_{e 2}\end{array}\right]=\mathbf{J}^{T}\left[\begin{array}{c}F_{e x} \\ F_{e y}\end{array}\right]$.

The equivalent closed-loop stiffnesses are obtained by computing the following equations:

$K_{c l 1}=\left[\begin{array}{l}1 \\ 0\end{array}\right]^{T} \mathbf{J}^{T}\left[\begin{array}{cc}K_{c l x} & 0 \\ 0 & K_{c l y}\end{array}\right] \mathbf{J}\left[\begin{array}{l}1 \\ 0\end{array}\right]$

$K_{c l 2}=\left[\begin{array}{l}0 \\ 1\end{array}\right]^{T} \mathbf{J}^{T}\left[\begin{array}{cc}K_{c l x} & 0 \\ 0 & K_{c l y}\end{array}\right] \mathbf{J}\left[\begin{array}{l}0 \\ 1\end{array}\right]$.

The global architecture of the controller is shown on Fig. 6 .

\section{Simulation results}

The aim of this section is to compare the performances of the control law defined in Section 5 with a classical linear impedance controller (see Fig. 7). Indeed, two simulations with different objectives are presented in the following subsections. These simulations have been obtained using the simulation model presented in Section 3. The first simulation have been set in order to compare the accuracy of the controllers while tracking desired position and pneumatic stiffness trajectories. The second simulation have been done to illustrate the controllers ability to reject disturbances with the desired stiffness behavior.

The chosen parameters for the simulation have been identified on the BirthSIM robot (Herzig et al., 2014, 2015) and are presented in Table 3.

It can be noticed that for all simulations presented in this paper, the control gains $C_{3}, C_{4}, K_{i 3}, C_{7}, C_{8}$, and $K_{i 8}$ which are not dependent of the closed-loop stiffness and damping tuning are set to the following constant values: $C_{3}=150, C_{4}=200, K_{i 3}=1500, C_{7}=150, C_{8}=200$, $K_{i 8}=1500$.

\subsection{Position and pneumatic stiffness tracking}

In this simulation, the objective is to compare the tracking performances of desired trajectories in position and pneumatic stiffness of both controllers. The following position trajectories have been chosen as $x_{c d}(t)=0.3+0.004 t$ and $y_{c d}(t)=-0.03+0.025 \sin \left(500 x_{c t}(t)\right)$. The pneumatic stiffness trajectory of each actuator has been defined as a succession of random steps. The transition between these steps have 


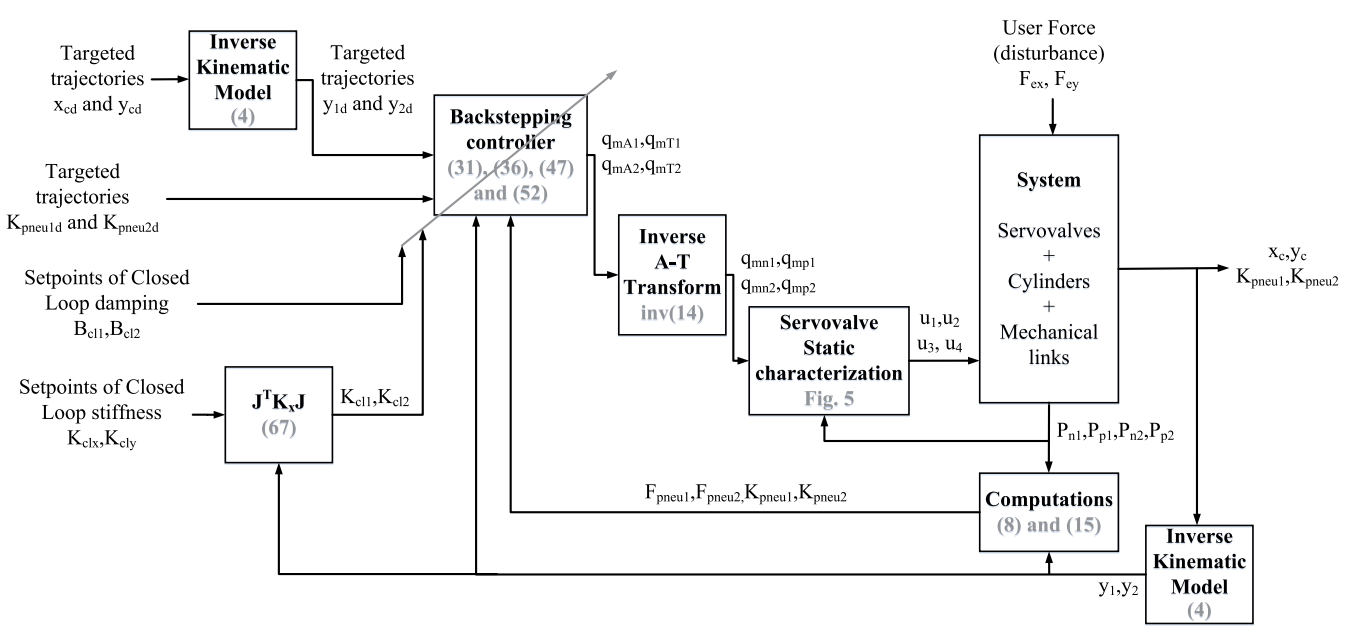

Fig. 6. Architecture of the Backstepping position and stiffness controller.

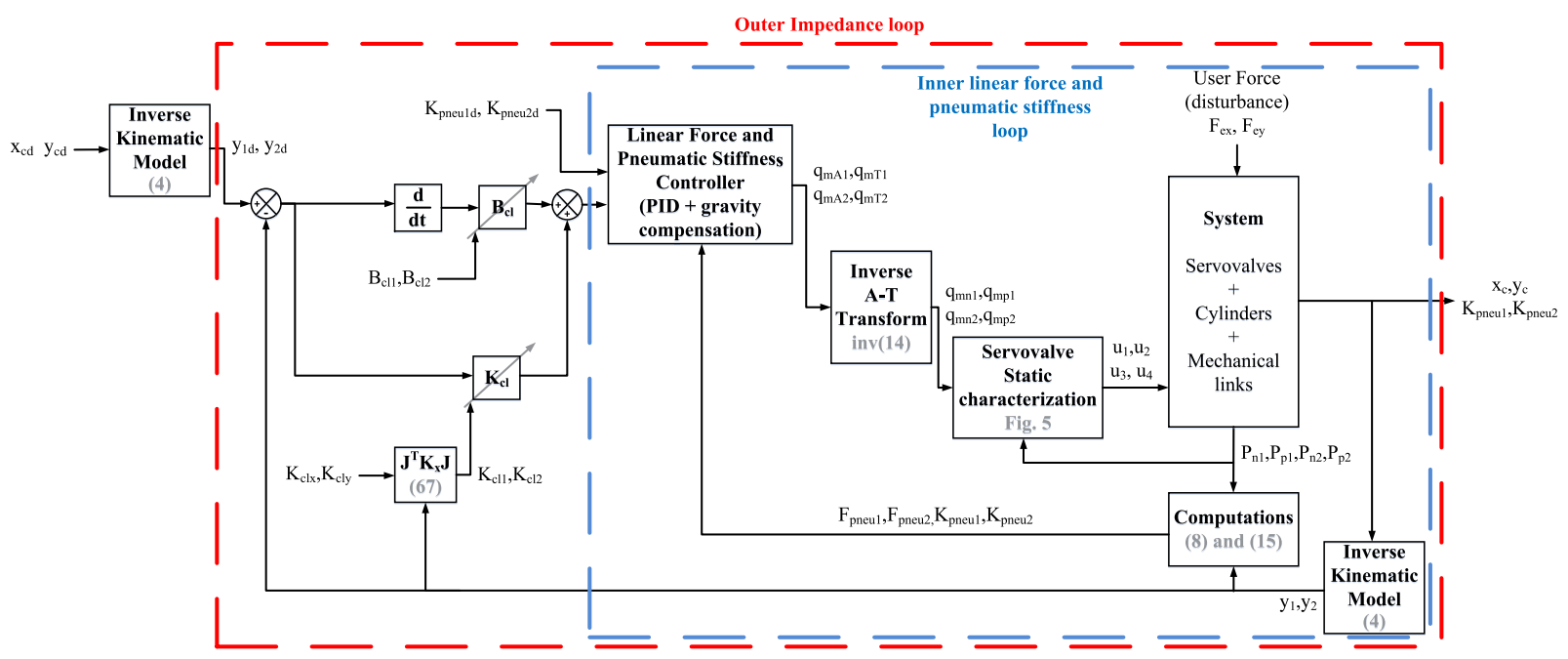

Fig. 7. Architecture of the linear impedance controller.

been smoothed with tanh function to respect the $C^{1}$ continuity for $K_{\text {pneud } 1}$

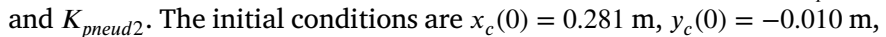
$K_{\text {pneu } 1}(0)=1091 \mathrm{~N} / \mathrm{m}$, and $K_{\text {pneu } 2}(0)=540 \mathrm{~N} / \mathrm{m}$. Fig. 8 shows the results of the position and pneumatic stiffness tracking. For this simulation, the closed-loop stiffnesses and dampings have been set to constant values $K_{c l x}(t)=2000 \mathrm{~N} / \mathrm{m}, K_{c l y}(t)=2000 \mathrm{~N} / \mathrm{m}, B_{c l 1}(t)=300 \mathrm{~N} . \mathrm{s} / \mathrm{m}$, and $B_{c l 2}(t)=400 \mathrm{~N} . \mathrm{s} / \mathrm{m}$.

Figs. 8(a) and 8(b) show that the targeted Cartesian positions and pneumatic stiffnesses are reached relatively quickly for both controllers. According to Fig. 8(c) the Backstepping controller is more accurate than the linear impedance controller, in particular on the Cartesian $y$ axis. Indeed, the Backstepping controller takes into account a part of the nonlinear inertial dynamic whereas the linear impedance controller does not. Concerning the tracking performances of pneumatic stiffnesses trajectories, both controller have similar accuracy. Finally Fig. 8(d) illustrates the motion of the end-effector in the Cartesian plane and the robot workspace.

\subsection{Disturbance rejection}

The aim of the second simulation is to show the behavior of the two DOF robot and the controller when submitted to a disturbance. Thus, the references of Cartesian positions and pneumatic stiffnesses have been set to constant values which are $x_{c d}(t)=0.350 \mathrm{~m}, y_{c d}(t)=-5 \times 10^{-3} \mathrm{~m}$,
$K_{\text {pneud } 1}=6000 \mathrm{~N} / \mathrm{m}$, and $K_{\text {pneud } 2}=3000 \mathrm{~N} / \mathrm{m}$. During the simulation, disturbance forces are applied on the robot end-effector. These forces denoted $F_{e x}$, and $F_{e y}$ are respectively applied along the Cartesian $x$ and $y$ axis. The two disturbances are repeated 6 times during the simulation with a $90^{\circ}$ phase shift between them. For the three first disturbances, the closed-loop dampings $B_{c l 1}$ and $B_{c l 2}$ are set respectively at 250 N.s/m and 500 N.s/m whereas the closed-loop stiffnesses vary gradually between each disturbance from $1500 \mathrm{~N} / \mathrm{m}$ up to $3000 \mathrm{~N} / \mathrm{m}$. Then, for the three last disturbances, the sequences of closed-loop stiffnesses are repeated whereas the closed-loop dampings are doubled. These settings are illustrated on Fig. 9(a).

According to Fig. 9(b), the behaviors expected for closed-loop stiffnesses and dampings tuning are successful. Indeed, increasing the closed-loop stiffness reduces the displacement due to a disturbance forces and increasing the damping increases the settling time of this displacement. Fig. 9(c) shows the position of the end-effector in Cartesian plane. On this figure, the first second which corresponds to the stabilization time has been removed. Finally, Fig. 9(d) illustrates the joint position. On this figure the expected joint positions $y_{1 \exp }$ and $y_{2 \exp }$, $x_{\text {cexp }}$ and $y_{\text {cexp }}$ are obtained by adding the estimated displacement due to perturbations to the targeted trajectories. Thus

$y_{1 \exp }=y_{d 1}+\frac{F_{e 1}}{K_{c l 1}} y_{2 e x p}=y_{d 2}+\frac{F_{e 2}}{K_{c l 2}}$
$x_{c e x p}=x_{c d}+\frac{F_{e x}}{K_{c l x}} \quad y_{c e x p}=y_{c d}+\frac{F_{e y}}{K_{c l y}}$. 

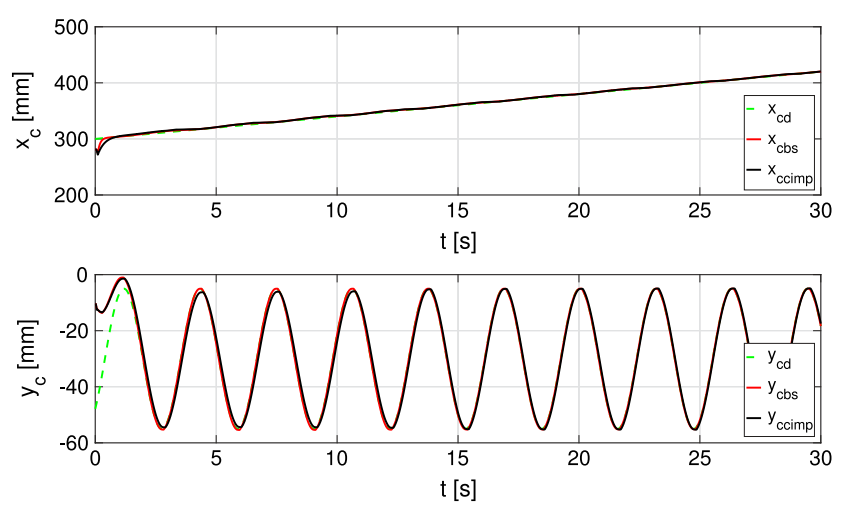

(a) Cartesian position tracking.
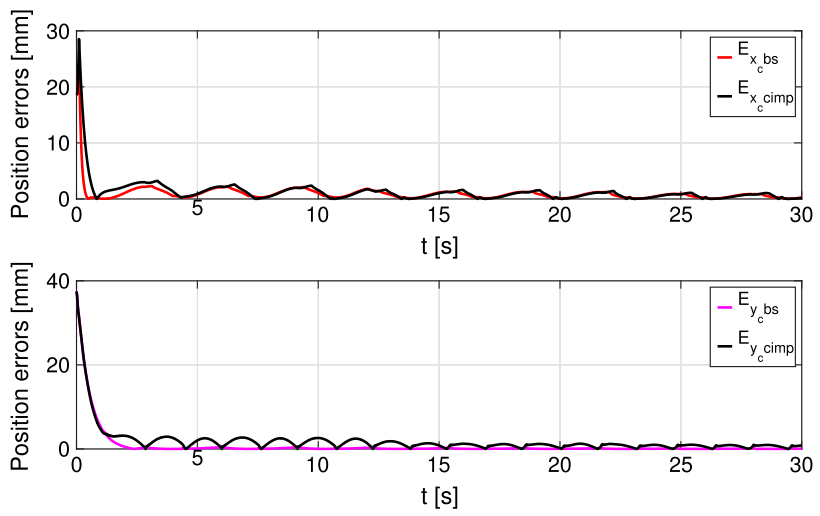

(c) Tracking errors.
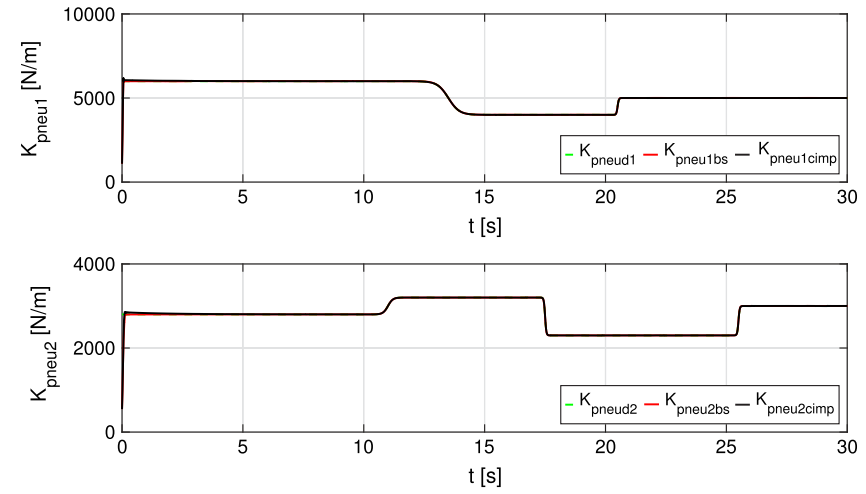

(b) Pneumatic stiffness tracking.

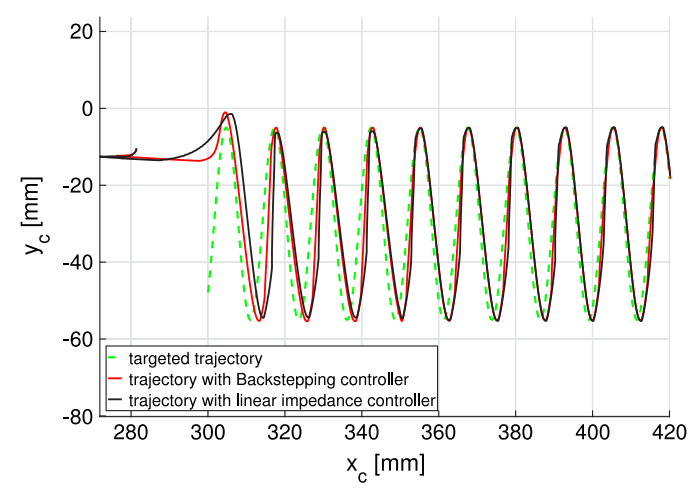

(d) End-effector trajectory in the Cartesian plane.

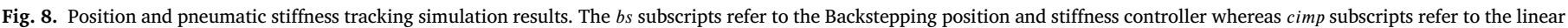
impedance controller.

This simulation shows that the performances of the closed loop stiffness tuning are similar for both controller. Table 4 compares the Cartesian closed-loop stiffnesses measured, denoted $K_{\text {clxm }}$ and $K_{\text {clym }}$ for the stiffness on $x$ and $y$ Cartesian axis respectively, with the Cartesian closed-loop stiffnesses set, denoted $K_{c l x}$ and $K_{c l y}$, for each direction of force disturbance. This table also gives the corresponding relative errors of Cartesian stiffnesses, denoted $E_{K_{c l x}}$ and $E_{K_{c l y}}$. According to the results, the performances to reject the disturbances with a desired closed loop stiffness are nearly the same for both controllers.

It can be noticed that even if the joint positions reach the expected positions, the Cartesian closed-loop stiffnesses are not necessary obtained. This phenomenon can be explained by two reasons, the first one is that the non-diagonal elements of the joint stiffness matrix have not been taken into account in the controller synthesis. The second reason is that the classical transformation used to compute the joint stiffness matrix from the Cartesian stiffness matrix (c.f. (67)) is not conservative, so this transformation is valid only when the robot is at its unloaded equilibrium. Some conservative transformations have been studied (Chen \& Kao, 2000) but these transformations need to measure or observe the disturbance force value. The results show that the bigger the position errors are, the bigger the Cartesian closed-loop stiffness errors are.

\section{Experimental results}

This section gives some experimental results to compare the two controllers presented in this paper. As in Section 7, this section will be divided into two subsections. The Section 8.1 gives the results for a reference position tracking. The Section 8.2 illustrates the behavior of the system when a disturbance force is applied.

\subsection{Position tracking}

For this experiment, the targeted trajectory is a circle in the Cartesian space. Indeed, the Cartesian coordinate of the end-effector references are defined as follows: $x_{c d}(t)=0.45+0.04 \cos (2 \pi t / 6)$ and $y_{c d}(t)=$ $0.025+0.04 \sin (2 \pi t / 6)$. The closed loop stiffnesses and dampings are set as $K_{c l x}=3000 \mathrm{~N} / \mathrm{m}, K_{c l y}=3000 \mathrm{~N} / \mathrm{m}, B_{c l 1}=300 \mathrm{~N} . \mathrm{s} / \mathrm{m}$ and $B_{c l 2}=300 \mathrm{~N} . \mathrm{s} / \mathrm{m}$. Fig. 10 shows the result for the position tracking for each controllers. Fig. 11 gives the position error.

According to those figures, the behavior expected on the $y$ axis is obtained. Indeed, the oscillations of the position errors due to the inertial dynamic can be observed. And for the $y$ axis, the Backstepping position and stiffness controller is more accurate than the impedance controller, as expected with the simulation results in Section 7.1. On the other hand, it can be noticed that a stick and slip effect occurred on the $x$ axis. Indeed, for both controllers, the horizontal cylinder piston is sometimes stuck by the friction. This phenomenon is due to the $2 \mathrm{~kg}$ load added to the end-effector. As shown on the Fig. 1 there is no linear guide to distribute the load on the end-effector. As a consequence, the cylinder rod is bending which increase a lot the frictions. To avoid that issue, a linear guide will be added to the cylinder 2 in the future. It can be noticed that the Coulomb friction model is not suitable to model the stick and slip phenomenon. To increase the accuracy of the model, a LuGre friction model could replace the Coulomb model (Armstrong-Hélouvry, Dupont, \& Canudas De Wit, 1994). As expected in the previous section, the performances of both controllers for position tracking on $x$ axis are equivalent, but the high peaks on the $x$ axis for the Backstepping controller position error show that the latest is more disturbed by the stick and slip phenomenon. 

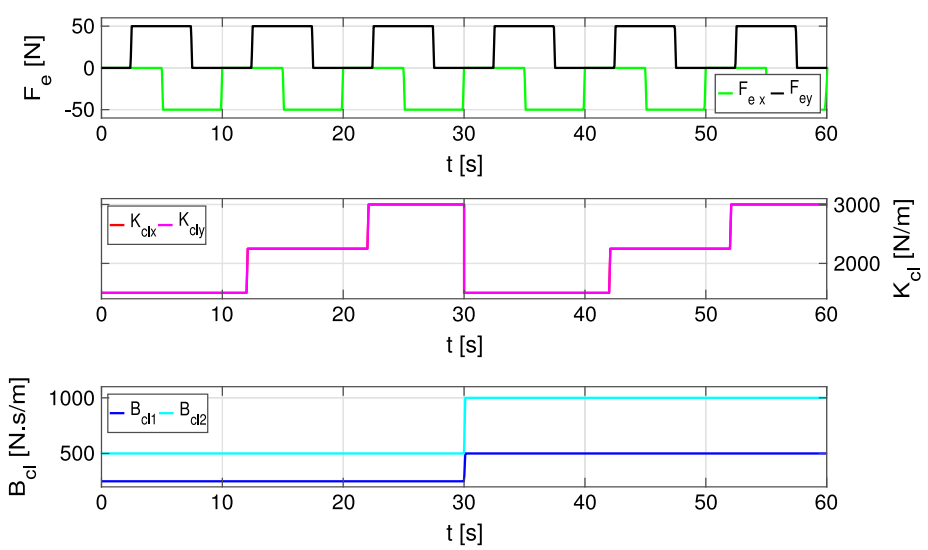

(a) Disturbance, closed-loop stiffnesses and closed-loop dampings.

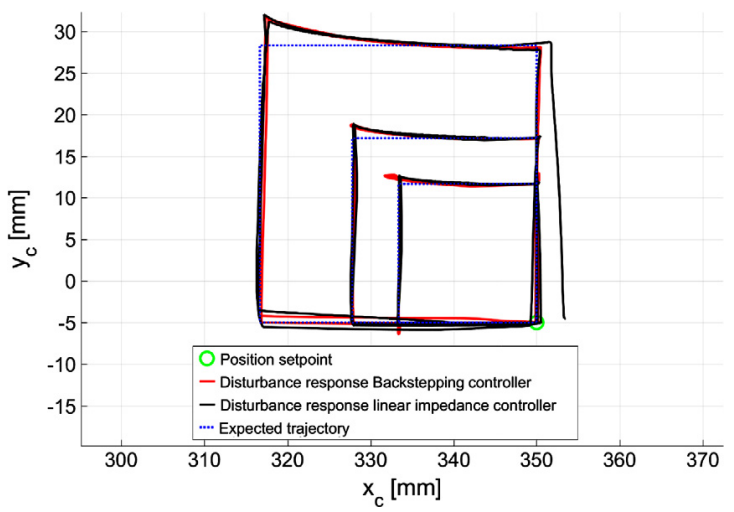

(c) End-effector trajectory in the Cartesian plane.
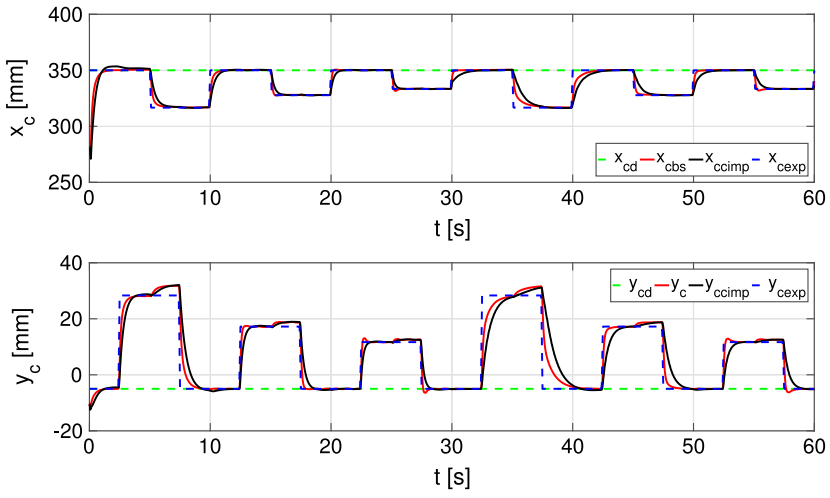

(b) Cartesian position.
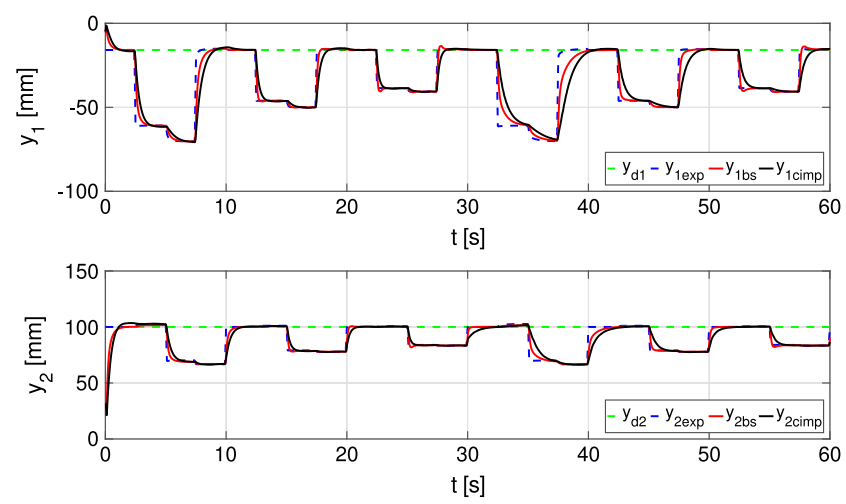

(d) Joint positions.

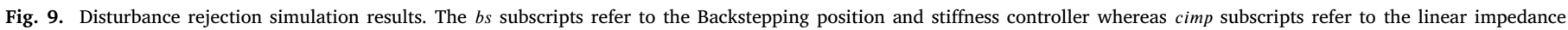
controller.
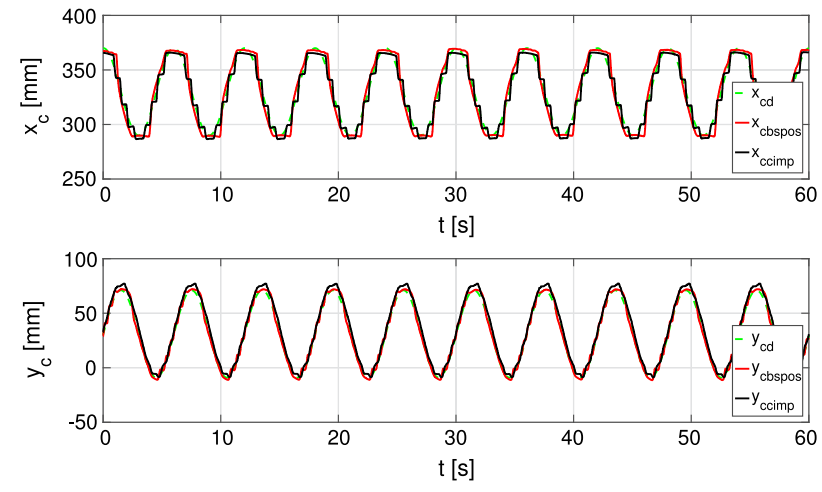

Fig. 10. Experimental Cartesian position tracking.

\subsection{Disturbance rejection}

As in Section 7.2, for this experiment, a constant position reference is set and some disturbance forces are applied on $x$ and $y$ axis. Those disturbances have been applied manually. A NANO25 ATI force and torque sensor have been added to the end-effector to measure the disturbance forces applied. Due to the difficulty to apply manually a constant force on both directions at the same time, it has been decided to keep the forces applied on the two directions separately. The experiment has been repeated 5 times for each controller at different position references.
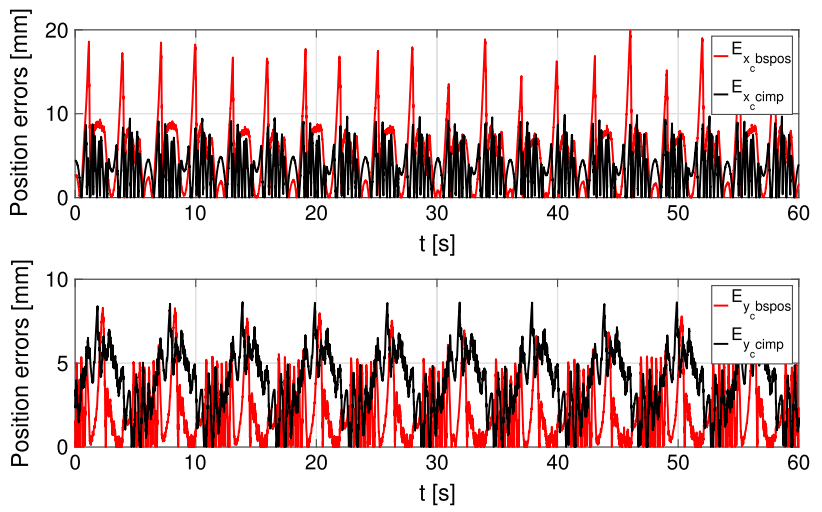

Fig. 11. Experimental tracking errors.

Figs. 12 and 13 illustrate one of the trials with Backstepping position and stiffness controller and one of the trials with the impedance controller respectively. Figs. 12(a) and 13(a) give the disturbance forces applied and the closed loop stiffness references for the two controllers respectively. Figs. 12(b) and 13(b) show the time response of endeffector Cartesian coordinates to the disturbance forces. These figures show that the two controllers reject the disturbance force as expected. The manually applied disturbance forces are noisy. Due to this noise, it is, unfortunately, impossible to conclude on the closed loop damping. It has to be noticed that the forces are applied directly to the 6 axis force 

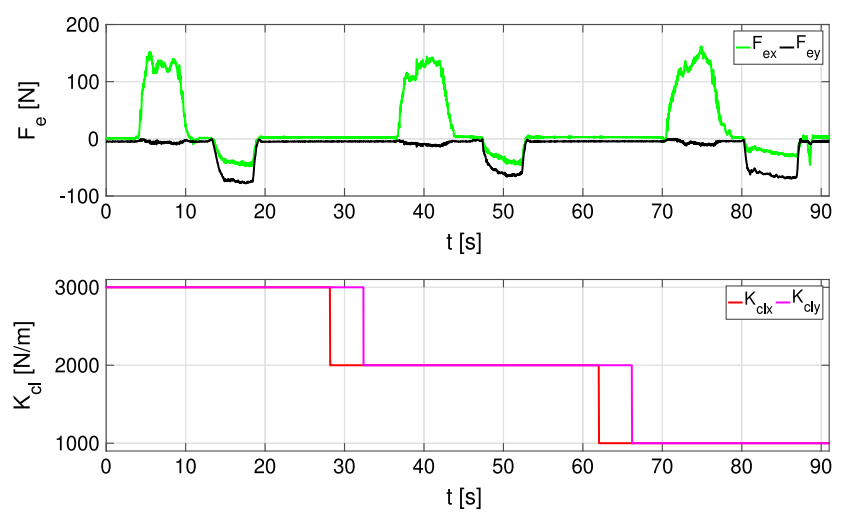

(a) Disturbance and closed-loop stiffnesses.
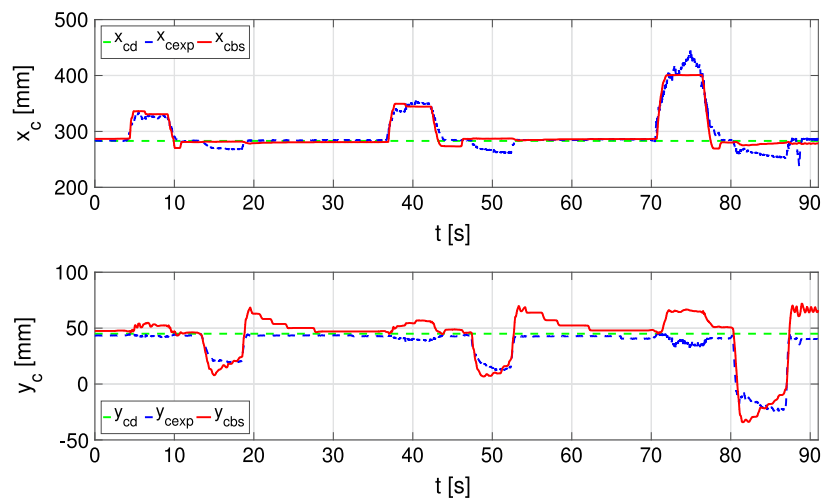

(b) Cartesian position.

Fig. 12. Disturbance rejection experimental results for the Backstepping controller.
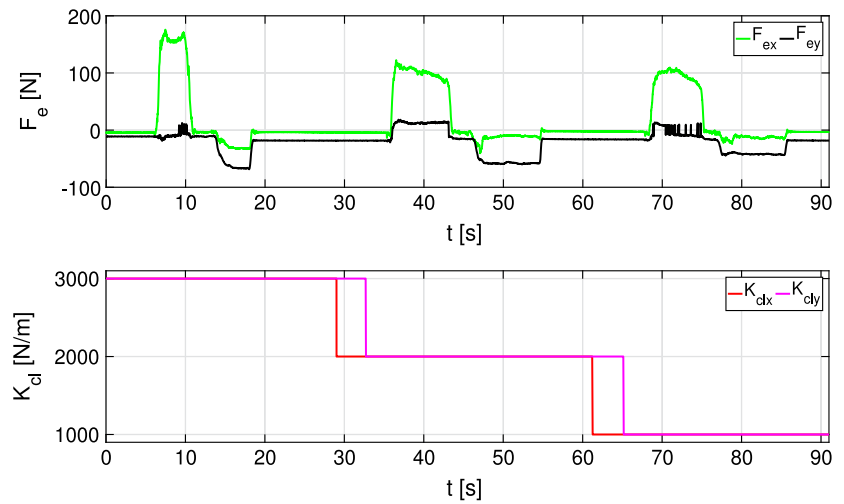

(a) Disturbance and closed-loop stiffnesses.
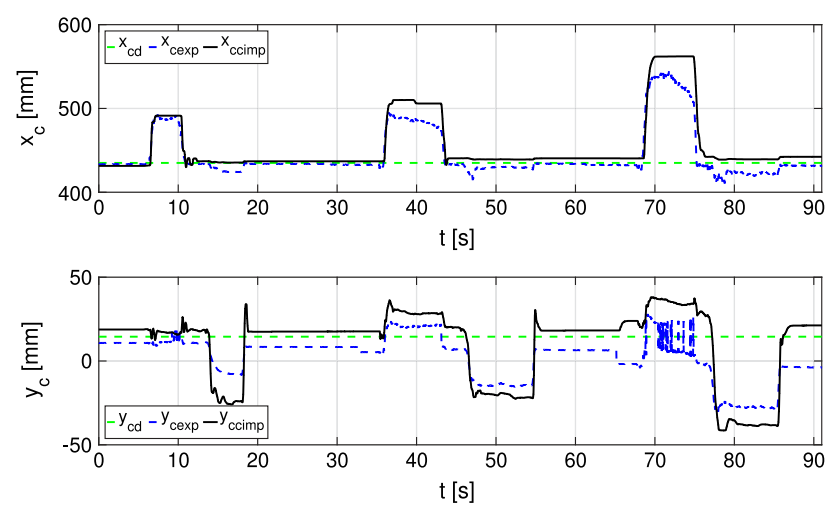

(b) Cartesian position.

Fig. 13. Disturbance rejection experimental results for the impedance controller.
Table 4

Cartesian closed-loop stiffnesses errors.

\begin{tabular}{|c|c|c|c|c|c|c|c|}
\hline \multirow{6}{*}{ 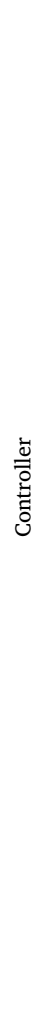 } & \multirow{3}{*}{ 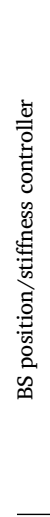 } & \multirow{3}{*}{ 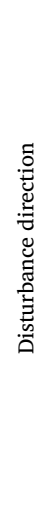 } & $\uparrow$ & $\begin{array}{l}K_{c l y}[N / m] \\
\Delta y_{c}[m m] \\
K_{c l y m}[N / m] \\
E_{K_{c l y}}[\%]\end{array}$ & $\begin{array}{l}1500 \\
33.08 \\
1511 \\
0.77\end{array}$ & $\begin{array}{l}2250 \\
22.29 \\
2243 \\
-0.30\end{array}$ & $\begin{array}{l}3000 \\
16.58 \\
3001 \\
0.04\end{array}$ \\
\hline & & & $\leftarrow$ & $\begin{array}{l}K_{c l x}[N / m] \\
\Delta x_{c}[m m] \\
K_{c l x m}[N / m] \\
E_{K_{c l x}}[\%] \\
\end{array}$ & $\begin{array}{l}1500 \\
-33 \\
1515 \\
1.01\end{array}$ & $\begin{array}{l}2250 \\
-22 \\
2273 \\
1.01\end{array}$ & $\begin{array}{l}3000 \\
-16.6 \\
3012 \\
0.40\end{array}$ \\
\hline & & & $\nwarrow$ & $\begin{array}{l}K_{c l y}[N / m] \\
\Delta y_{c}[m m] \\
K_{c l y m}[N / m] \\
E_{K_{c l y}}[\%] \\
K_{c l x}[N / m] \\
\Delta x_{c}[m m] \\
K_{c l x m}[N / m] \\
E_{K_{c l x}}[\%] \\
\end{array}$ & $\begin{array}{l}1500 \\
36.75 \\
1361 \\
-9.30 \\
1500 \\
-32.8 \\
1524 \\
1.63 \\
\end{array}$ & $\begin{array}{l}2250 \\
23.64 \\
2115 \\
-6.00 \\
2250 \\
-22.1 \\
2262 \\
0.55\end{array}$ & $\begin{array}{l}3000 \\
17.5 \\
2857 \\
-4.76 \\
3000 \\
-16.4 \\
3049 \\
1.63\end{array}$ \\
\hline & $\ddot{ \pm}$ & & $\uparrow$ & $\begin{array}{l}K_{c l y}[N / m] \\
\Delta y_{c}[m m] \\
K_{c l y m}[N / m] \\
E_{K_{c l y}[\%]}[\%]\end{array}$ & $\begin{array}{l}1500 \\
33.7 \\
1483 \\
-1.09 \\
\end{array}$ & $\begin{array}{l}2250 \\
22.29 \\
2243 \\
-0.30 \\
\end{array}$ & $\begin{array}{l}3000 \\
16.66 \\
3001 \\
0.04\end{array}$ \\
\hline & 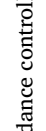 & 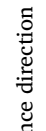 & $\leftarrow$ & $\begin{array}{l}K_{c l x}[N / m] \\
\Delta x_{c}[m m] \\
K_{c l x m}[N / m] \\
E_{K_{c l x}}[\%]\end{array}$ & $\begin{array}{l}1500 \\
-33 \\
1515 \\
1.01\end{array}$ & $\begin{array}{l}2250 \\
-22 \\
2273 \\
1.01\end{array}$ & $\begin{array}{l}3000 \\
-16.6 \\
3012 \\
0.40\end{array}$ \\
\hline & 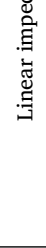 & 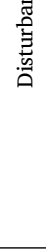 & $\nwarrow$ & $\begin{array}{l}K_{c l y}[N / m] \\
\Delta y_{c}[m m] \\
K_{c l y m}[N / m] \\
E_{K_{c l y}}[\%] \\
K_{c l x}[N / m] \\
\Delta x_{c}[m m] \\
K_{c l x m}[N / m] \\
E_{K_{c l x}}[\%]\end{array}$ & $\begin{array}{l}1500 \\
37.06 \\
1349 \\
-10.06 \\
1500 \\
-32.8 \\
1524 \\
1.63\end{array}$ & $\begin{array}{l}2250 \\
23.85 \\
2096 \\
-6.83 \\
2250 \\
-22 \\
2273 \\
1.01\end{array}$ & $\begin{array}{l}3000 \\
17.5 \\
2857 \\
-4.76 \\
3000 \\
-16.4 \\
3049 \\
1.63\end{array}$ \\
\hline
\end{tabular}

Table 5

Summary of the experimental results for the closed loop stiffness tuning.

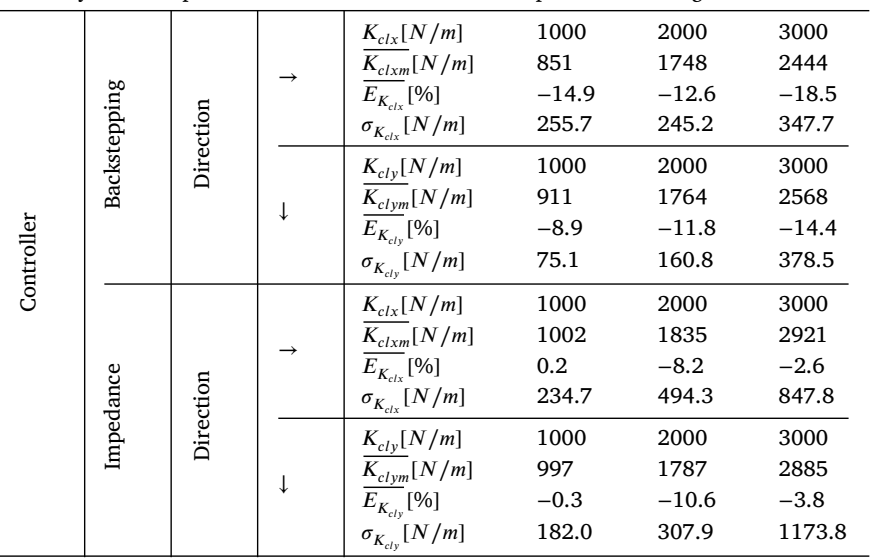

and torque sensor. The $x$ component of the disturbance force measured when a force on $y$ axis is applied is due to the grabbing of the sensor.

Tables B.6 and B.7 give the results for each trial for the Backstepping position and stiffness controller and the impedance controller respectively. A summary of the results is given on Table 5. Where $K_{c l x}$ and $K_{c l y}$ are the stiffness references, $\overline{K_{c l x m}}$ and $\overline{K_{c l y m}}$ are the average values of the stiffnesses computed from the five trials, $\overline{E_{K_{c l x}}}$ and $\overline{E_{K_{c l y}}}$ are the average values of the stiffness errors computed from the five trials and finally $\sigma_{K_{c l x}}$ and $\sigma_{K_{c l y}}$ are the standard deviations of the stiffnesses computed from the five trials.

Table 5 shows that the Impedance controller is, in average more accurate than the Backstepping position and stiffness controller for the closed loop stiffness tuning. On the other hand, the standard deviation of the closed loop stiffnesses obtained is smaller for the Backstepping controller than for the impedance controller. That means that the 
Table B.6

Experimental results for closed loop stiffness tuning with the Backstepping position and stiffness controller.

\begin{tabular}{|c|c|c|c|c|c|c|c|c|c|c|c|c|c|c|c|}
\hline Trial & 1 & & & 2 & & & 3 & & & 4 & & & 5 & & \\
\hline$K_{c l x}[N / m]$ & 1000 & 2000 & 3000 & 1000 & 2000 & 3000 & 1000 & 2000 & 3000 & 1000 & 2000 & 3000 & 1000 & 2000 & 3000 \\
\hline$\overline{F_{e x}}[N]$ & 128.1 & 108 & 136 & 66.5 & 114.3 & 92.4 & 97 & 95.7 & 126.6 & 134 & 125.2 & 129.3 & 45.7 & 91 & 105.8 \\
\hline$\overline{\Delta x_{c}}[\mathrm{~mm}]$ & 119.2 & 65.8 & 47.8 & 94 & 63.9 & 46.1 & 119.5 & 69.2 & 49 & 117.7 & 62.9 & 49.4 & 87.2 & 46.9 & 48.9 \\
\hline$K_{c l x m}[N / m]$ & 1074 & 1640.6 & 2845.8 & 707.8 & 1788.1 & 2005.3 & 811.3 & 1383.1 & 2585.2 & 1138.5 & 1988.8 & 2619.1 & 523.9 & 1938.3 & 2162.6 \\
\hline$E_{K_{c l x}}[\%]$ & 7.4 & -18 & -5.1 & -29.2 & -10.6 & -33.2 & -18.9 & -30.8 & -13.8 & 13.9 & -0.6 & -12.7 & -47.6 & -3.1 & -27.9 \\
\hline$K_{c l y}^{c l x}[N / m]$ & 1000 & 2000 & 3000 & 1000 & 2000 & 3000 & 1000 & 2000 & 3000 & 1000 & 2000 & 3000 & 1000 & 2000 & 3000 \\
\hline$\overline{F_{e y}}[N]$ & -56.8 & -68.2 & -74.4 & -63.5 & -79.3 & -77.2 & -71.4 & -73.1 & -76.8 & -63.1 & -60.8 & -74.3 & -62.2 & -63 & -67.6 \\
\hline$\overline{\Delta y_{c}}[\mathrm{~mm}]$ & -72.8 & -45 & -37.9 & -68.4 & -45.2 & -31 & -73.4 & -37.5 & -26.4 & -66.9 & -34.5 & -28.4 & -66.9 & -34.2 & -23.7 \\
\hline$K_{c l y m}[N / m]$ & 780.1 & 1513.7 & 1964.4 & 928.6 & 1753.7 & 2494.1 & 971.9 & 1951.2 & 2912.5 & 943.7 & 1762.8 & 2613.5 & 929.1 & 1839.4 & 2855.2 \\
\hline$E_{K_{c l y}}[\%]$ & -22 & -24.3 & -34.5 & -7.1 & -12.3 & -16.9 & -2.8 & -2.4 & -2.9 & -5.6 & -11.9 & -12.9 & -7.1 & -8 & -4.8 \\
\hline
\end{tabular}

Table B.7

Experimental results for closed loop stiffness tuning with the impedance controller.

\begin{tabular}{|c|c|c|c|c|c|c|c|c|c|c|c|c|c|c|c|}
\hline Trial & 1 & & & 2 & & & 3 & & & 4 & & & 5 & & \\
\hline$K_{c l x}[N / m]$ & 1000 & 2000 & 3000 & 1000 & 2000 & 3000 & 1000 & 2000 & 3000 & 1000 & 2000 & 3000 & 1000 & 2000 & 3000 \\
\hline$\overline{F_{e x}}[N]$ & 151.4 & 181.3 & 185.3 & 154.2 & 169.5 & 227.3 & 100 & 108.9 & 119.9 & 98.4 & 102.8 & 160.2 & 116.1 & 147.2 & 142.9 \\
\hline$\overline{\Delta x_{c}}[\mathrm{~mm}]$ & 129.1 & 78.8 & 64.9 & 117.2 & 72.5 & 53.7 & 124.1 & 86.2 & 64.5 & 126.6 & 72.8 & 56.4 & 123.4 & 79 & 50.7 \\
\hline$K_{c l x m}[N / m]$ & 1172.7 & 2301.6 & 2854.4 & 1315.7 & 2338.5 & 4234.7 & 805.8 & 1263.7 & 1858.9 & 777.1 & 1410.9 & 2841.8 & 940.7 & 1862.1 & 2817.3 \\
\hline$E_{K_{c l x}}[\%]$ & 17.3 & 15.1 & -4.9 & 31.6 & 16.9 & 41.2 & -19.4 & -36.8 & -38 & -22.3 & -29.5 & -5.3 & -5.9 & -6.9 & -6.1 \\
\hline$K_{c l y}^{c x}[N / m]$ & 1000 & 2000 & 3000 & 1000 & 2000 & 3000 & 1000 & 2000 & 3000 & 1000 & 2000 & 3000 & 1000 & 2000 & 3000 \\
\hline$\overline{F_{e y}}[N]$ & -75.1 & -64.4 & -84.4 & -65 & -79.6 & -83.6 & -65.1 & -68.3 & -64.1 & -41.8 & -58 & -64.4 & -56.8 & -51.9 & -55.3 \\
\hline $\overrightarrow{\Delta y_{c}}[\mathrm{~mm}]$ & -65.4 & -30 & -20.2 & -53.5 & -38.5 & -20.4 & -76.8 & -41.8 & -25.9 & -52.3 & -34.9 & -38.3 & -58.5 & -36.4 & -27.7 \\
\hline$K_{\text {clym }}[N / m]$ & 1147.9 & 2145.8 & 4170.7 & 1216.1 & 2069.7 & 4092.8 & 847.4 & 1633.9 & 2478.4 & 800.2 & 1660.9 & 1680.3 & 971.4 & 1424.9 & 2000.5 \\
\hline$E_{K_{c l y}}[\%]$ & 14.8 & 7.3 & 39 & 21.6 & 3.5 & 36.4 & -15.3 & -18.3 & -17.4 & -20 & -17 & -44 & -2.9 & -28.8 & -33.3 \\
\hline
\end{tabular}

Backstepping controller has a better repeatability on the stiffness tuning than the impedance controller.

\section{Conclusions and future works}

In this study, a two DOF pneumatic robot design and model are proposed. The assumptions and transformations to synthesize a position controller with the Backstepping method are provided. Then a strategy of gain tuning, which leads to a closed-loop stiffness and damping control, is presented. Finally, the performances of this controller are illustrated with some simulation and experimental results. These performances are discussed and compared to a classical linear impedance controller with gravity compensation. This simulation approach is generally used to design a controller for pneumatic or hydraulic actuator before experimental implementation (Smaoui, Brun, \& Thomasset, 2006). According to the simulation result, the Backstepping position controller with the closed loop stiffness tuning strategy is more accurate for the position tracking than the linear impedance controller. Concerning, the performances for the closed loop stiffness tuning, they are similar for the two controllers. The experimental results have confirmed those expectations. Indeed the Backstepping position and stiffness controller is more accurate for the position tracking in particular on the $y$ axis, but this controller is also more sensitive to stick and slip phenomenon. These results are in agreement with the experimental results given by Herzig, Moreau, Leleve, and Pham (2016) for a 1 DOF pneumatic robot.

The performance of the presented controller can be improved by adding a linear guide to the horizontal cylinder to avoid the bending of the cylinder rod. Another way to improved the accuracy of the model and the controller for the position tracking is adding a LuGre model which is suitable to model the stick and slip phenomenon.

On the other hand, a way to improve the closed-loop stiffness accuracy of the controller is to use a stiffness matrix transformation which is conservative. In order to do that without adding a force sensor, the disturbances have to be observed. So adding a robust observer for the external disturbance force could be an interesting improvement the presented controller.

Finally, from the experiment results given in this paper, the damping tuning cannot be analyzed. Indeed, in the future, the robot will be coupled to an antagonist robot which will apply the disturbance forces. This robot will allow disturbance forces without noise to be applied, but also to study the behavior of the controller to sinusoidal disturbances and then analyze the dynamic disturbance rejection.

\section{Acknowledgments}

This work was funded by the ANR French National Research Agency [ANR-12-MONU-0006]. This work was partially supported by the Engineering and Physical Sciences Research Council [EP/N03211X/2 Morphological computation of perception and action].

Appendix A. Calculation of the first pneumatic effort virtual input time derivative

$$
\begin{array}{r}
\dot{F}_{\text {pneu } 1}^{*}=\frac{M v_{2} d_{6}^{2} \cos \theta_{5}+M\left(y_{2}+k_{2}\right) d_{6}^{2} \dot{\theta}_{5}}{d_{6}^{4} \cos ^{2} \theta_{5}}\left[-g d_{6} \sin \theta_{1}\right. \\
\left.+2 v_{1} v_{2}+\left(y_{2}+k_{2}\right)\left(a_{d 1}+\left(C_{5}^{2}-1\right) z_{5}-\left(C_{5}+C_{6}\right) z_{6}\right)\right] \\
+\frac{M\left(y_{2}+k_{2}\right)}{d_{6}^{2} \cos \theta_{5}}\left[-g d_{6} \dot{\theta}_{1} \cos \theta_{1}+2\left(\frac { d _ { 6 } } { y _ { 2 } + k _ { 2 } } \left(g \sin \theta_{1}\right.\right.\right. \\
\left.\left.-\frac{d_{6} \cos \theta_{5}}{M\left(y_{2}+k_{2}\right)}\left(F_{f 1}+F_{\text {pext } 1}-F_{\text {pneu } 1}\right)\right)-\frac{v_{1} v_{2}}{y_{2}+k_{2}}\right) v_{2} \\
+2 v_{1}\left(\frac{F_{\text {pneu } 2}-F_{\text {pext } 2}-F_{f 2}}{M}+g \cos \theta_{1}\right. \\
\left.+\frac{4\left(y_{2}+k_{2}\right)\left(y_{1}+k_{1}\right)^{2} v_{1}^{2}}{4 d_{3}^{2} d_{6}^{2}-\left(\left(y_{1}+k_{1}\right)^{2}-d_{3}^{2}-d_{6}^{2}\right)^{2}}\right)+v_{2}\left(a_{d 1}\right. \\
\left.+\left(C_{5}^{2}-1\right) z_{5}-\left(C_{5}+C_{6}\right) z_{6}\right)+\left(y_{2}+k_{2}\right)\left(j_{d 1}\right. \\
+\left(C_{5}^{2}-1\right)\left(z_{6}-C_{5} z_{5}\right)-\left(C_{5}+C_{6}\right)\left(\frac { d _ { 6 } } { y _ { 2 } + k _ { 2 } } \left(g \sin \theta_{1}\right.\right. \\
\left.\left.\left.\left.-\frac{d_{6} \cos \theta_{5}}{M\left(y_{2}+k_{2}\right)}\left(F_{f 1}+F_{p e x t 1}-F_{\text {pneu } 1}\right)\right)-\frac{v_{1} v_{2}}{y_{2}+k_{2}}\right)\right)\right]
\end{array}
$$

Appendix B. Experimental results for closed loop stiffness tuning tables

Tables B.6 and B.7 give the results for the closed loop stiffness tuning for each trials for the Backstepping position and stiffness controller and 
the impedance controller respectively. In those tables $\overline{F_{e x}}, \overline{F_{e y}}$, are the average value of the force on $x$ axis and the average value of the force on $y$ axis, respectively, when the disturbance is applied on the relative direction. $\overline{\Delta x_{c}}$ and $\overline{\Delta y_{c}}$ are the average values of the displacement of the end-effector due to the disturbance on respectively $x$ and $y$ axis, when the relative disturbance is applied. Then the Cartesian closedloop stiffnesses measured, denoted $K_{c l x m}$ and $K_{c l y m}$ and the relative errors of Cartesian stiffnesses, denoted $E_{K_{c l x}}$ and $E_{K_{c l y}}$, are computed as follows:

$$
\begin{gathered}
K_{c l x m}=\frac{\overline{F_{e x}}}{\overline{\Delta x_{c}}} \quad K_{c l y m}=\frac{\overline{F_{e y}}}{\overline{\Delta y_{c}}} \\
E_{K_{c l x}}=\frac{K_{c l x m}-K_{c l x}}{K_{c l x}} \quad E_{K_{c l y}}=\frac{K_{c l y m}-K_{c l y}}{K_{c l y}} .
\end{gathered}
$$

\section{References}

Abry, F., Brun, X., Sesmat, S., Bideaux, E., \& Ducat, C. (2015). Electropneumatic cylinder backstepping position controller design with real-time closed-loop stiffness and damping tuning. IEEE Transactions on Control Systems Technology, PP(99). http: //dx.doi.org/10.1109/TCST.2015.2460692.

Andersen, B. (1967). The analysis and design of pneumatic systems. Wiley.

Armstrong-Hélouvry, B., Dupont, P., \& Canudas De Wit, C. (1994). A survey of models, analalysis tools and compensation methods for control of machines with friction. Automatica, 30(7), 1083-1138.

Bobrow, J., \& McDonell, B. (1998). Modeling, identification, and control of a pneumatically actuated, force controllable robot. IEEE Transactions on Robotics and Automation, 14(5), 732-742. http://dx.doi.org/10.1109/70.720349.

Chen, S.-F., \& Kao, I. (2000). Conservative congruence transformation for joint and cartesian stiffness matrices of robotic hands and fingers. International Journal of Robotics Research, 19(9), 835-847. http://dx.doi.org/10.1177/02783640022067201. http://arxiv.org/abs/http://ijr.sagepub.com/content/19/9/835.full.pdf + html.

Freeman, R., \& Kokotovic, P. (1993). Design of softer robust nonlinear control laws. Automatica, 29(6), 1425-1437. http://dx.doi.org/10.1016/0005-1098(93)90007-G.

Hayati, S. (1986). Hybrid position/force control of multi-arm cooperating robots. In Robotics and automation. Proceedings. 1986 IEEE international conference on: Vol. 3. (pp. 82-89). http://dx.doi.org/10.1109/ROBOT.1986.1087650.

Herzig, N., Moreau, R., Leleve, A., \& Pham, M. (2016). Stiffness control of pneumatic actuators to simulate human tissues behavior on medical haptic simulators. In 2016 IEEE international conference on advanced intelligent mechatronics, AIM (pp. 15911597). http://dx.doi.org/10.1109/AIM.2016.7576997.
Herzig, N., Moreau, R., \& Redarce, T. (2014). A new design for the birthsim simulator to improve realism. In Engineering in medicine and biology society, 2014. EMBC 2014. Annual international conference of the IEEE (pp. 2065-2068).

Herzig, N., Moreau, R., Redarce, T., Abry, F., \& Brun, X. (2015). Non linear position and closed loop stiffness control for a pneumatic actuated haptic interface: The Birthsim. In 2015 IEEE/RSJ international conference on intelligent robots and systems, IROS (pp. 1612-1618).

Hogan, N. (1987). Stable execution of contact tasks using impedance control. In Robotics and automation. Proceedings. 1987 IEEE international conference on: Vol. 4. (pp. 10471054). http://dx.doi.org/10.1109/ROBOT.1987.1087854.

Huang, Y., Vanderborght, B., Van Ham, R., Wang, Q., Van Damme, M., Xie, G., \& Lefeber, D. (2013). Step length and velocity control of a dynamic bipedal walking robot with adaptable compliant joints. IEEE/ASME Transactions on Mechatronics, 18(2), 598-611. http://dx.doi.org/10.1109/TMECH.2012.2213608.

Khalil, W., \& Kleinfinger, J. (1986). A new geometric notation for open and closed-loop robots. In Robotics and automation. Proceedings. 1986 IEEE international conference on: Vol. 3. IEEE.

Paul, R. P. (1982). Robot manipulators: Mathematics, programming, and control. MIT Press.

Salisbury, J. (1980). Active stiffness control of a manipulator in cartesian coordinates. In Decision and control including the symposium on adaptive processes, 1980 19th IEEE conference on (pp. 95-100). http://dx.doi.org/10.1109/CDC.1980.272026.

Shearer, J. L. (1956). Study of pneumatic processes in the continuous control of motion with compresses air part I. Transaction of the ASME (American Society of Mechanical Engineers), 78, 233-242.

Shen, X., \& Goldfarb, M. (2007). Simultaneous force and stiffness control of a pneumatic actuator. Journal of Dynamic Systems Measurement and Control-Transactions, 129(4), 425-434.

Smaoui, M., Brun, X., \& Thomasset, D. (2006). Systematic control of an electropneumatic system: integrator backstepping and sliding mode control. IEEE Transactions on Control Systems Technology, 14, 905-913. http://dx.doi.org/10.1109/TCST.2006.880183.

Taheri, B., Case, D., \& Richer, E. (2014). Force and stiffness backstepping-sliding mode controller for pneumatic cylinders. Mechatronics, IEEE/ASME Transactions on, 19(6), 1799-1809. http://dx.doi.org/10.1109/TMECH.2013.2294970.

Van Ham, R., Sugar, T., Vanderborght, B., Hollander, K., \& Lefeber, D. (2009). Compliant actuator designs. IEEE Robotics Automation Magazine, 16(3), 81-94. http://dx.doi.org/ 10.1109/MRA.2009.933629.

Yao, B., \& Tomizuka, M. (2001). Adaptive robust control of \{MIMO\} nonlinear systems in semi-strict feedback forms. Automatica, 37(9), 1305-1321. http://dx.doi.org/10. 1016/S0005-1098(01)00082-6. 